\title{
A Phosphorylated Histone H2A Variant Displays Properties of Chromatin Insulator Proteins in Drosophila
}

\author{
James R. Simmons ${ }^{1 \#}$, Ran An ${ }^{1 \#}$, Bright Amankwaa ${ }^{1}$, Shannon Zayac ${ }^{1}$, Justin Kemp ${ }^{1}$, and Mariano Labrador ${ }^{1 *}$ \\ ${ }^{1}$ Department of Biochemistry and Cellular and Molecular Biology, The University of Tennessee, Knoxville, TN 37996, USA \\ * Author for correspondence (labrador@utk.edu) \\ \# These authors contributed equally to this work.
}

\begin{abstract}
Chromatin insulators are responsible for mediating long-range interactions between enhancers and promoters throughout the genome and align with the boundaries of topologically associating domains (TADs). Here, we demonstrate an interaction between proteins that associate with the gypsy insulator and the phosphorylated histone variant $\mathrm{H} 2 \mathrm{Av}(\mathrm{\gamma H} 2 \mathrm{Av})$, a marker of DNA double strand breaks. Gypsy insulator components colocalize with $\mathrm{\gamma H} 2 \mathrm{Av}$ throughout the genome. Mutation of insulator components prevents stable $\mathrm{H} 2 \mathrm{Av}$ phosphorylation in polytene chromatin. Phosphatase inhibition strengthens the association between insulator components and $\mathrm{\gamma H} 2 \mathrm{Av}$ and rescues $\mathrm{y} 2 \mathrm{Av}$ localization in insulator mutants. We also show that $\gamma \mathrm{H} 2 \mathrm{Av}$ is a component of insulator bodies, and that phosphatase activity is required for insulator body dissolution after recovery from osmotic stress. We further demonstrate a tight association between $\mathrm{\gamma H} 2 \mathrm{Av}$ and TAD boundaries. Together, our results indicate a novel mechanism linking insulator function with a histone $\mathrm{H} 2 \mathrm{~A}$ variant and with genome stability.
\end{abstract}

\section{Introduction}

A highly orchestrated 3D-genome organization is necessary for the proper function and survival of eukaryotic cells. The resulting higher-order chromosome structure in this organization is driven through the establishment of long-range interactions between different regions of the chromosome. These interactions create domains that may be restricted from interacting with each other, and are based on specific interactions between chromatin-binding proteins (van Berkum et al., 2010, Rao et al., 2014, Fudenberg et al., 2016). Chromatin insulators represent a class of protein/DNA complexes associated to specific sequences in the genome that work through two general functions: to restrict communication between enhancers and promoters through physical separation into different genomic domains and to prevent the spread of heterochromatin into euchromatic regions of the genome (Harrison et al., 1989, Geyer and Corces, 1992, Bell et al., 1999, Schoborg and Labrador, 2014, Ozdemir and Gambetta, 2019). The presence of insulators in the genome is conserved among eukaryotes, with the CTCF insulator being the only known insulator regulating the human genome (Heger and Wiehe, 2014). Insulators have recently been described to help establish the boundaries of topologically associating domains (TADs) and are often found enriched at TAD boundaries (Van Bortle et al., 2014, Fudenberg et al., 2016).

Drosophila melanogaster has an array of different insulator complexes, with each complex being recruited to different sequences in the genome (Bushey et al., 2009, Negre et al., 2010). Insulators were first described in fruit flies with the discovery that the scs and scs' sequences function as boundary elements, inhibiting chromosomal position effects (Kellum and Schedl, 1991). Another insulator site, located within the gypsy retrotransposon, has been thoroughly characterized for its ability to regulate transcription of neighboring genes (Geyer and Corces, 1992).
A number of gypsy retrotransposons are present throughout the Drosophila genome (Hoskins et al., 2015), and insertion or transposition of gypsy to a new locus may interrupt local transcriptional activity and chromatin dynamics (Jack et al., 1991, Geyer and Corces, 1992). Insulator proteins are recruited to gypsy through a 460-bp sequence composed of 12 binding sites for Suppressor of Hairy Wing (Su(Hw)) (Geyer and Corces, 1992). The $\mathrm{Su}(\mathrm{Hw})$ protein contains an amino-terminal tandem array of a dozen $\mathrm{C}_{2} \mathrm{H}_{2}$ zinc finger domains (Kim et al., 1996) and a carboxyterminal leucine zipper domain (Harrison et al., 1993) that is essential for insulator activity through interactions with other insulator proteins (Melnikova, Kostyuchenko, Molodina, et al., 2018). Su(Hw) specifically recruits one of the many isoforms of Modifier of mdg4 (Mod(mdg4)67.2) through interactions between the carboxy-terminal domain of $\mathrm{Su}(\mathrm{Hw})$ and carboxy-terminal acidic domain that is unique to the gypsy-binding isoform of Mod(mdg4) (Ghosh et al., 2001) and through interactions between the amino-terminal end of $\mathrm{Su}(\mathrm{Hw})$ with the glutamine-rich domain of Mod(mdg4)67.2 (Melnikova, Kostyuchenko, et al., 2017). Centrosomal Protein 190 (CP190), another essential component of the gypsy insulator (Pai et al., 2004), was originally described through its activity during the cell cycle, dissociating from chromatin during mitotic prophase and localizing to the centrosome (Ooegema et al., 1995). In chromatin, CP190 is found as an essential part of different insulator complexes (Bushey et al., 2009) and is recruited to the gypsy insulator through interactions with Mod(mdg4)67.2 (Pai et al., 2004) and the amino terminal domain of HIPP1 (HP1 and insulator partner protein 1) (Melnikova et al., 2019). HIPP1 is the most recently described member of the gypsy insulator complex (Alekseyenko et al., 2014) and functions to stabilize the interaction between $\mathrm{Su}(\mathrm{Hw})$ and CP190, but is not required for insulator activity or transcriptional regulation (Glenn and Geyer, 2019, Melnikova et al., 2019).

Insulator activity in gypsy is promoted by factors such as the interchromosomal EAST protein (Golovnin et al., 2015, Melnikova, Shapovalov, et al., 2017) and the Chromatin-linked adapter for MSL 
proteins (CLAMP) (Bag et al., 2019), while activity is negatively regulated by RNA-binding proteins Rumpelstiltskin (King et al., 2014) and Shep (Chen et al., 2019). Insulator-binding proteins in Drosophila can form aggregates known as insulator bodies (Gerasimova and Corces, 1998). The role of these bodies in genome organization has been debated, and functions for insulator bodies have been proposed from genome organization hubs to passive storage centers for insulator proteins (Gerasimova et al., 2000, Labrador and Corces, 2002, Golovnin et al., 2012). Previous work in our lab has demonstrated a role for insulator bodies in the cellular response to osmotic stress, with insulator proteins leaving chromatin and forming bodies in the nucleoplasm as the environment becomes more hypertonic (Schoborg et al., 2013).

Of the gypsy insulator proteins, $\mathrm{Su}(\mathrm{Hw})$ is perhaps the bestcharacterized. Mutation of $s u(H w)$ is associated with female infertility (Klug et al., 1968, Harrison et al., 1993, Baxley et al., 2011, Hsu et al., 2020) and alters the cell's response to DNA damage (Lankenau et al., 2000). Outside of the gypsy insulator complex, $\mathrm{Su}(\mathrm{Hw})$ binds many sites alone or in conjunction with either Mod(mdg4)67.2 or CP190 (Adryan et al., 2007, Kuhn-Parnell et al., 2008, Bushey et al., 2009, Negre et al., 2010, Soshnev et al., 2012, Baxley et al., 2017).

Apart from its role in chromatin insulator activity, $\mathrm{Su}(\mathrm{Hw})$ is also a transcriptional repressor in Drosophila oogenesis (Baxley et al., 2011) and regulates the expression of neuronal genes in tissues outside the nervous system, including the ovary (Soshnev et al., 2013). Mutation of $s u(H w)$ leads to defects in the formation of ring canals between nurse cells, preventing the proper transport of maternal morphogens during ovary development (Hsu et al., 2015). Ovaries in $s u(H w)$ mutants also exhibit malformed microtubule organizing centers and mislocalization of Gurken, a morphogen normally sequestered to one end of the oocyte to direct axis determination in embryos (Hsu et al., 2020). Similar to its role in oogenesis, $\mathrm{Su}(\mathrm{Hw})$ is critical for spermatogenesis through its activity as a transcriptional repressor in somatic cyst cells in the male germline (Duan and Geyer, 2018).

$\mathrm{Su}(\mathrm{Hw})$ also participates in the DNA damage response, possibly as part of the search for homologous sequences during homologous recombination (Lankenau et al., 2000). A role for insulators in homologous recombination-based DNA repair has been well established with mammalian CTCF, which is recruited to sites of DNA double strand breaks (DSBs) (Han et al., 2017, Hilmi et al., 2017, Lang et al., 2017). Of clinical relevance, mutation of CTCF in humans is associated with various forms of cancer (Docquier et al., 2005, Kemp et al., 2014, Canela et al., 2017, Guo et al., 2018). One of the first steps in the cellular response to DSBs is the phosphorylation of a variant of $\mathrm{H} 2 \mathrm{~A}$ known as $\mathrm{H} 2 \mathrm{AX}$ in mammalian systems and H2Av in Drosophila (Rogakou et al., 1998, Baldi and Becker, 2013). H2AX is phosphorylated by ATM (Ataxiatelangiectasia-mutated) kinase and DNA-dependent protein kinase (DNA-PK) in response to ionizing radiation (Stiff et al., 2004) and by ATR (ataxia telangiectasia and Rad3-related) kinase after cells experience replication-induced genotoxic stress (Ward and Chen, 2001). Phosphorylation of H2AX leads to recruitment of numerous proteins involved in the DNA damage response (Sirbu and Cortez, 2013). Upon resolution of the DSB, H2AX is dephosphorylated primarily by PP2A (Chowdhury et al., 2005). H2Av, the sole Drosophila H2A variant (Baldi and Becker, 2013), combines the activities of mammalian $\mathrm{H} 2 \mathrm{AX}$ and $\mathrm{H} 2 \mathrm{AZ}$. Like $\mathrm{H} 2 \mathrm{AX}, \mathrm{H} 2 \mathrm{Av}$ is phosphorylated in response to DNA damage (Madigan et al., 2002), and like $\mathrm{H} 2 \mathrm{AZ}$, phosphorylation of $\mathrm{H} 2 \mathrm{Av}$ is involved in transcriptional regulation through activation of poly(ADP-ribose) polymerase 1 (PARP1) (Kotova et al., 2011). Our laboratory previously demonstrated an accumulation of phosphorylated $\mathrm{H} 2 \mathrm{Av}$ $(\gamma \mathrm{H} 2 \mathrm{Av})$ signal in the ovaries of $s u(\mathrm{Hw})$ mutants and the presence of chromosomal aberrations in actively dividing larval neuroblasts lacking $\mathrm{Su}(\mathrm{Hw})$, suggesting a significant connection between $\mathrm{Su}(\mathrm{Hw})$ activity and genome stability (Hsu et al., 2020). Furthermore, disruption of Mei-41/ATR, a kinase responsible for phosphorylating $\mathrm{H} 2 \mathrm{Av}$ among other targets upon DNA damage (LaRocque et al., 2007), partially rescues the defective oogenesis phenotype associated with mutation of $s u(\mathrm{Hw})$ (Hsu et al., 2020). While insulator-binding proteins have been described for their role in genome organization and regulation in Drosophila, the mechanisms linking their activity to DNA repair remain elusive.

In this work, we show that $\mathrm{\gamma H} 2 \mathrm{Av}$ is present at $\mathrm{Su}(\mathrm{Hw})$-binding sites throughout the genome, including at gypsy retrotransposons and that mutation of several gypsy insulator components disrupts normal H2Av phosphorylation patterns. We show that $\mathrm{\gamma H} 2 \mathrm{Av}$ is a component of insulator bodies formed under osmotic stress and that dephosphorylation of $\mathrm{\gamma H} 2 \mathrm{Av}$ is required for efficient dissolution of these bodies during recovery. Chromatin immunoprecipitation (ChIP) experiments reveal extensive genomewide colocalization between $\mathrm{Su}(\mathrm{Hw})$ and $\mathrm{\gamma H} 2 \mathrm{Av}$ and enrichment for both at TAD boundaries. This association also extends to insulator function as flies doubly heterozygous for His $2 A v^{810}$ and mutant alleles of $s u(H w)$ showed a partial rescue of phenotypes for yellow ${ }^{2}$ and cut $^{6}$, two gypsy insulator induced mutations. Collectively, these findings point to a model in which $\mathrm{\gamma H} 2 \mathrm{Av}$ works with insulators to coordinate genome function and genome-wide responses to genotoxic stress.

\section{Materials and Methods}

\section{Fly stocks and husbandry}

All stocks were maintained on a standard cornmeal agar fly food medium supplemented with yeast at $20^{\circ} \mathrm{C}$; crosses were carried out at $25^{\circ} \mathrm{C}$. The following stocks are maintained in our lab and were originally obtained from Victor Corces (Emory University): $y^{2} w^{1} c t^{6}$; cp190 $1931-2 / T M 6 B, T b^{1}, y^{2} w^{1} c t^{6} ; c p 190^{P 11} / T M 6 B, T b^{1}, y^{2} w^{1} c t^{6} ; w^{1118}$; $s u(H w)^{V} / T M 6 B, T b^{1}, y^{2} w^{1} c t^{6} ; \bmod (\operatorname{mdg} 4)^{u 1} / T M 6 B, T b^{1}$. The stock $w^{1118}$; PBac(RB)su(Hw)e04061/TM6B, b $^{1}$ was obtained from the Bloomington Drosophila stock center (BDSC: 18224). These remaining stocks were provided by our lab: OR, $y^{2} w^{1} c t^{6}$;

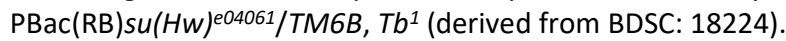

\section{Antibodies}

Rabbit polyclonal IgG antibodies against Su(Hw), Mod(mdg4)67.2, and CP190 were previously generated by our lab (Wallace et al., 
2010, Schoborg et al., 2013). A rat polyclonal IgG antibody against $\mathrm{Su}(\mathrm{Hw})$ generated by our lab was also used. Antibody against the phosphorylated form of H2Av (UNC93-5.2.1) (Lake et al., 2013) was obtained from the Developmental Studies Hybridoma Bank, created by the NICHD of the NIH and maintained at The University of lowa, Department of Biology, lowa City, IA 52242. These antibodies were all diluted 1:1 in glycerol (Fisher Scientific, BP2291 , lot 020133 ) and used at a final dilution of 1:200. Secondary antibodies were all diluted $1: 1$ in glycerol and used at a final dilution of 1:200. The following secondary antibodies were used in this study: Alexa Fluor 594 goat anti-rabbit (Invitrogen, A-111037, lot 2079421), Alexa Fluor 488 donkey anti-rabbit (Invitrogen, A21206, lot 1834802), Alexa Fluor 488 goat anti-guinea pig (Invitrogen, A-11073, lot 84E1-1), Texas red donkey anti-rat (Jackson ImmunoResearch Laboratories, 712-075-150), and Alexa Fluor 488 goat anti-mouse (Invitrogen, A-11001, lot 1858182).

\section{Immunostaining of larval tissues}

Wandering third instar larvae were dissected in PBS. Tissues were immediately placed into fixative (4\%, para-formaldehyde (Alfa Aesar, 43368, lot N13E011), 50\% glacial acetic acid (Fisher Scientific, A38-212, lot 172788)) on a coverslip for one minute. Samples were squashed by lowering a slide on top of the sample then turning it over, placing it between sheets of blotting paper, and hitting the coverslip firmly with a small rubber mallet. Slides were dipped in liquid nitrogen, coverslips were removed, and samples were incubated in blocking solution (3\% powdered nonfat milk in PBS + 0.1\% IGEPAL CA-630 (Sigma-Aldrich, 18896, lot 1043)) for 10 minutes at room temperature. The slides were dried and incubated with primary antibodies overnight at $4^{\circ} \mathrm{C}$ in a box humidified with wet paper towels. The next day, slides were washed twice in PBS + 0.1\% IGEPAL CA-630 before incubation with secondary antibodies for three hours in the dark at room temperature. Slides were washed twice in PBS + 0.1\% IGEPAL CA630 , treated with DAPI solution of $0.5 \mu \mathrm{g} / \mathrm{mL}$ (ThermoFisher, D1306) for one minute, and washed one more time in PBS alone.

Samples were mounted with Vectashield antifade mounting medium (Vector Laboratories, H-1000, lot ZF0409) and coverslips were sealed with clear nail polish. All microscopy for immunostaining was performed on a wide-field epifluorescent microscope (DM6000 B; Leica Microsystems) equipped with a 100x/1.35 NA oil immersion objective and a charge-coupled device camera (ORCA-ER; Hamamatsu Photonics). Image acquisition was performed using SimplePCI (v6.6; Hamamatsu Photonics). Image manipulation was performed in FIJI (Schindelin et al., 2012); all contrast adjustments are linear. Images were further processed in Adobe Photoshop CS5 Extended, Version $12.0 \times 64$. Figures were assembled in Adobe Illustrator CS5, Version 15.0.0. Statistical analyses were performed in GraphPad Prism version 8.0.0 (224) (GraphPad Software, San Diego, CA).

\section{Immunostaining of S2 cells}

For normal control conditions, S2 cells were incubated in insect medium (HyClone SFX-Insect Cell Culture Media; Fisher Scientific, SH3027802) supplemented with penicillin (50 units $/ \mathrm{mL}$ ) and streptomycin $(50 \mu \mathrm{g} / \mathrm{mL})($ Gibco, 15070063$)$ at $25^{\circ} \mathrm{C}$. To induce osmotic stress, the istonic media was replaced with hypertonic media supplemented with $250 \mathrm{mM} \mathrm{NaCl}$ (Fisher Scientific, BP358212). Cells were treated in this hypertonic stress media for 30 minutes. Coverslips were pretreated with pure ethanol (Decon Labs, 2716) and coated with concanavalin A (Sigma-Aldrich, C5275) to help S2 cells adhere to the glass surface. Cells were pipetted onto treated coverslips and were allowed to spread and adhere for 30 minutes. After treatment, cells were fixed (4\%, paraformaldehyde, $50 \%$ acetic acid) for 10 minutes at room temperature, followed by three washes with PBS buffer. Fixed cells were permeabilized with $0.2 \%$ Triton X-100 (Fisher Scientific, BP151, lot 014673) for five minutes then washed twice with PBS buffer. Cells were incubated in blocking solution (3\% powdered nonfat milk in PBS + 0.1\% IGEPAL CA-630) for 10 minutes at room temperature. Primary antibodies were diluted in blocking solution and samples were incubated in antibody solution overnight at $4^{\circ} \mathrm{C}$ in a box humidified with wet paper towels. Unbound antibodies were washed off three times with PBST buffer (0.1\% Triton-X 100). Secondary antibody incubation, DAPI staining, and mounting were performed as described above.

\section{Okadaic acid treatment}

For the isotonic control samples, S2 cells were cultured in HyClone SFX-Insect media as above and incubated in $50 \mathrm{nM}$ okadaic acid (Sigma-Aldrich, 09381) for 30 minutes. The hypertonic samples were obtained by shifting S2 cells from isotonic insect media to hypertonic conditions as described above and incubating for 25 minutes. After this, the hypertonic media was supplemented with $50 \mathrm{nM}$ okadaic acid and cells were incubated for five more minutes. The isotonic recovery sample was obtained by first inducing hypertonic stress for thirty minutes, including okadaic acid for the final five minutes as above, then washing out the hypertonic media twice with isotonic media containing $50 \mathrm{nM}$ okadaic acid. Cells were incubated in isotonic recovery media with okadaic acid for thirty minutes. Control samples were collected throughout the process following the same protocol without addition of okadaic acid. For the polytene chromosome example, salivary glands were dissected from wandering third instar larvae and incubated in 50 $\mathrm{nM}$ okadaic acid for 30 minutes before fixation and squashing.

\section{Fluorescence intensity and colocalization analysis}

Images were analyzed for the amount of each protein (i.e. the intensity of each channel) using a macro script in FIJI (Schindelin et al., 2012). The DAPI channel was used to automatically generate non-biased ROIs for each cell, which were then manually curated for extra precision. A rolling-ball background subtraction algorithm was used for all images. Intensity measurements were made using the measure function. Numerous images of polytene 
chromosomes were collected from each salivary gland squash. All acquisition parameters were kept constant between slides within each experiment. Colocalization was quantified using the Coloc2 plugin in FIJI. This analysis uses the Costes method (Costes et al., 2004) to determine appropriate thresholds for each channel. Results are reported in terms of Pearson's Correlation Coefficient (PCC) (Pearson, 1895), which ranges from -1 for perfect anticorrelation to +1 for perfect correlation (Dunn et al., 2011). Another metric of colocalization is the Manders' Colocalization Coefficients (Manders et al., 1992, Manders et al., 1993, Adler and Parmryd, 2010) for each channel - this relates how much of the signal in the green channel overlaps with signal in the red channel (M1) and how much of the signal in the red channel overlaps with signal in the green channel (M2). M1 and M2 may vary from 0 , representing no overlap between signals, to 1 , representing total overlap.

\section{ChIP-seq and TAD data analysis}

The following NCBI publicly available ChIP-Seq datasets were used for the genome wide comparisons: SRX1299942 (yH2Av SRA (Li et al., 2016)), SRX046654 (Su(Hw) SRA (Chen et al., 2012)), SRX186113 (Mod(mdg4)67.2 SRA (Matzat et al., 2012)), SRX2638361 (CP190 SRA (Jox et al., 2017)), SRX2638363 (CTCF SRA (Jox et al., 2017)), and SRX511131 (HIPP1 SRA (Alekseyenko et al., 2014)). The sequencing data was uploaded to the Galaxy web platform, and the public server at usegalaxy.org was used to analyze the data (Afgan et al., 2018). Briefly, the FastQ datasets from NCBI were mapped with Bowtie2 to produce BAM files (Langmead and Salzberg, 2012). Duplicate and unmapped reads were filtered out with SAM tools. Peaks were called with Model-based analysis of ChIP-Seq (MACs) (Zhang et al., 2008). We used SeqMINER version 1.3.4 for the downstream plotting analysis (Ye et al., 2011, Zhan and Liu, 2015).

To compare the distribution of $\mathrm{\gamma H} 2 \mathrm{Av}$ with that of nucleosomal $\mathrm{H} 2 \mathrm{Av}$ we used the high-resolution distribution of homotypic and heterotypic Drosophila H2Av nucleosomes from S2 cells obtained from a previous study (Weber et al., 2010). FastQ datasets from paired-end reads from native micrococcal nuclease-digested chromatin, enriched in homotypic H2Av (SRX019957 (Weber et al., 2010)) or heterotypic H2Av (SRX019953 (Weber et al., 2010)), were mapped with Bowtie2 to produce BAM files mapped to the dm6 Drosophila genome. We applied Bamcoverage to BAM files to generate $\mathrm{H} 2 \mathrm{Av}$ Hom and H2Av Het bigwig files. SeqMINER was used to generate displays of the distribution of mean read density profiles (tags / $50 \mathrm{bp}$ ), using BED files to provide reference coordinates ( $\pm 10,000 \mathrm{bp}$ ). Bigwig files were generated by applying Bamcoverage to BAM files and peak profiles were visualized with the IGV genome browser (igv.org/app/) (Robinson et al., 2011), using Drosophila dm6 as the reference genome. The genomic distribution of TADs in the Drosophila genome (Ramirez et al., 2018) was used to produce a BED file generated with all genomic $1 \mathrm{~Kb}$ fragments containing a TAD boundary at the center.

\section{Phenotypic analysis}

Documentation of $y^{2}$ and $c t^{6}$ phenotypes was performed using a stereomicroscope (MZ16 FA; Leica Microsystems) equipped with a CCD color camera (DFC420; Leica Microsystems). A 150 Watt white light source (KL 1500 LCD; Leica Microsystems) set to a color temperature of $3,000 \mathrm{~K}$ was used for illumination. Male flies were selected soon after eclosion and aged for five days at $25^{\circ} \mathrm{C}$ before imaging. All images within each tissue set were collected with the same parameters using Leica Application Suite (Version 2.4.0 R1; Leica Microsystems). Abdomen images were recorded with a gamma correction of 0.5. Image analysis was performed in FIJI (Schindelin et al., 2012). Intensity of the darkest region within the fifth abdominal tergite was measured using a circular ROI with radius of 15 pixels $(=0.045 \mathrm{~mm}$ ) (Figure 8$)$. Intensity values from abdomens were inverted before analysis so that darker pigmentation provided a higher score. Regions of abdomens that reflected the light source were excluded from analysis. Wing areas were measured in FIJI (Schindelin et al., 2012) using the entire translucent area of the wing (except for the alula, which was sometimes lost in sample preparation) as the ROI.

\section{Results}

H2Av Phosphorylation Is Correlated with gypsy Insulator Components Genome-wide

Beyond its canonical insulator functions, the Drosophila insulator protein $\mathrm{Su}(\mathrm{Hw})$ has been implicated in transcriptional regulation and is required for development of testes and ovaries (Soshnev et al., 2013, Duan and Geyer, 2018). Mutation of su(Hw) interferes with normal DNA damage repair (Lankenau et al., 2000) and lack of $\mathrm{Su}(\mathrm{Hw})$ protein results in chromosomal aberrations in developing neuroblasts (Hsu et al., 2020). While these results point to a role in maintaining genome stability, the mechanistic link between $\mathrm{Su}(\mathrm{Hw})$ and DNA repair remains uncharacterized. To investigate this possibility, we performed immunostaining of polytene chromosomes from salivary glands of third instar larvae using an antibody directed against the phosphorylated form of $\mathrm{H} 2 \mathrm{Av}$ $(\gamma \mathrm{H} 2 \mathrm{Av})$, a marker of DNA double strand breaks (Madigan et al., 2002). This procedure reveals a close association between $\mathrm{Su}(\mathrm{Hw})$ and $\mathrm{\gamma H} 2 \mathrm{Av}$ throughout each polytene chromosome arm. Close inspection shows $\mathrm{\gamma H} 2 \mathrm{Av}$ in nearly all of the $\mathrm{Su}(\mathrm{Hw})$ bands (Figure $1 A)$. Linescans of the $2 R$ polytene chromosome show strong covariance in the fluorescent intensity between $\mathrm{\gamma H} 2 \mathrm{Av}$ and $\mathrm{Su}(\mathrm{Hw})$ (Figure 1A). Likewise, analysis of the immunostaining signal using the entire polytene genome shows a significant colocalization between $\mathrm{\gamma H} 2 \mathrm{Av}$ and $\mathrm{Su}(\mathrm{Hw})$ (Figure $1 \mathrm{~A}$ ). Quantification of colocalization of the two immunostaining signals is reported using Pearson's Correlation Coefficient (PCC), which describes the covariance between the two signals, with positive numbers describing direct correlation between signal intensities, negative numbers representing anti-correlation of the signals, and zero representing no correlation between signals (i.e. random covariance) (Adler and Parmryd, 2010, Dunn et al., 2011). Colocalization is also reported using Manders' overlap coefficient (MOC), which describes the amount of one signal in an image that overlaps with signal from the other channel (Manders et al., 1993). 
bioRxiv preprint doi: https://doi.org/10.1101/2021.02.23.432395; this version posted February 23, 2021. The copyright holder for this preprint (which was not certified by peer review) is the author/funder. All rights reserved. No reuse allowed without permission.

A

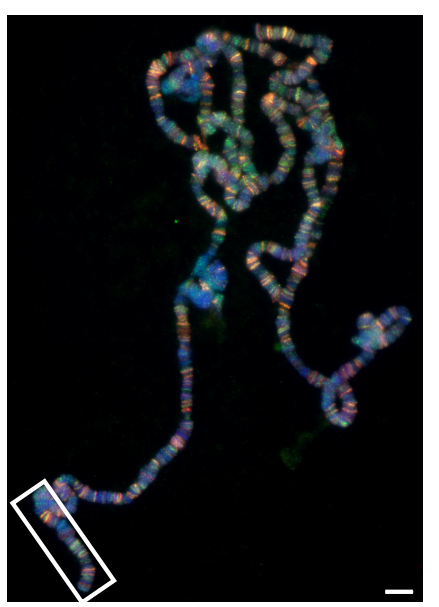

DAPI

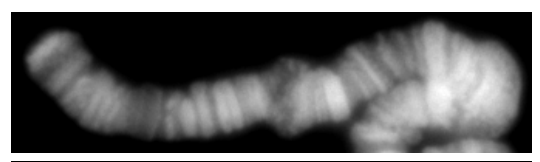

$\mathrm{Su}(\mathrm{Hw})$
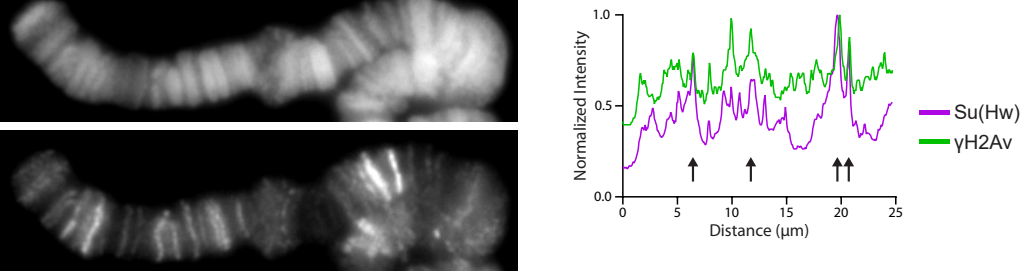

pH2Av

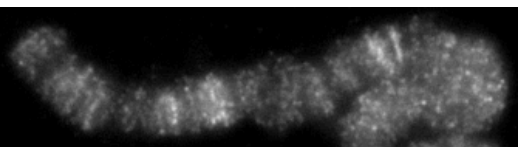

$\mathrm{Su}(\mathrm{Hw})$

$\mathrm{YH} 2 \mathrm{Av}$
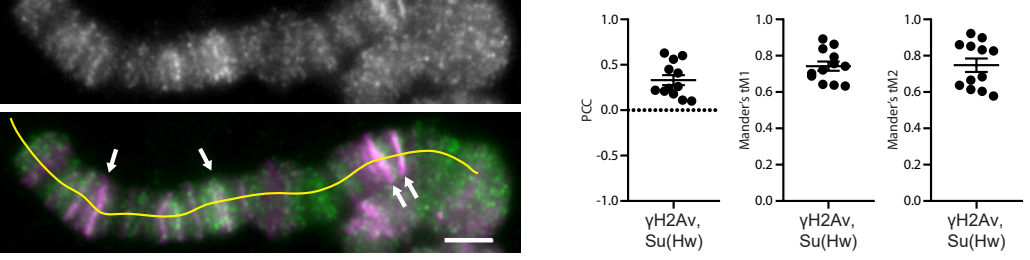

$\mathrm{Su}(\mathrm{Hw}), \mathrm{yH} 2 \mathrm{Av}$, DAPI

B
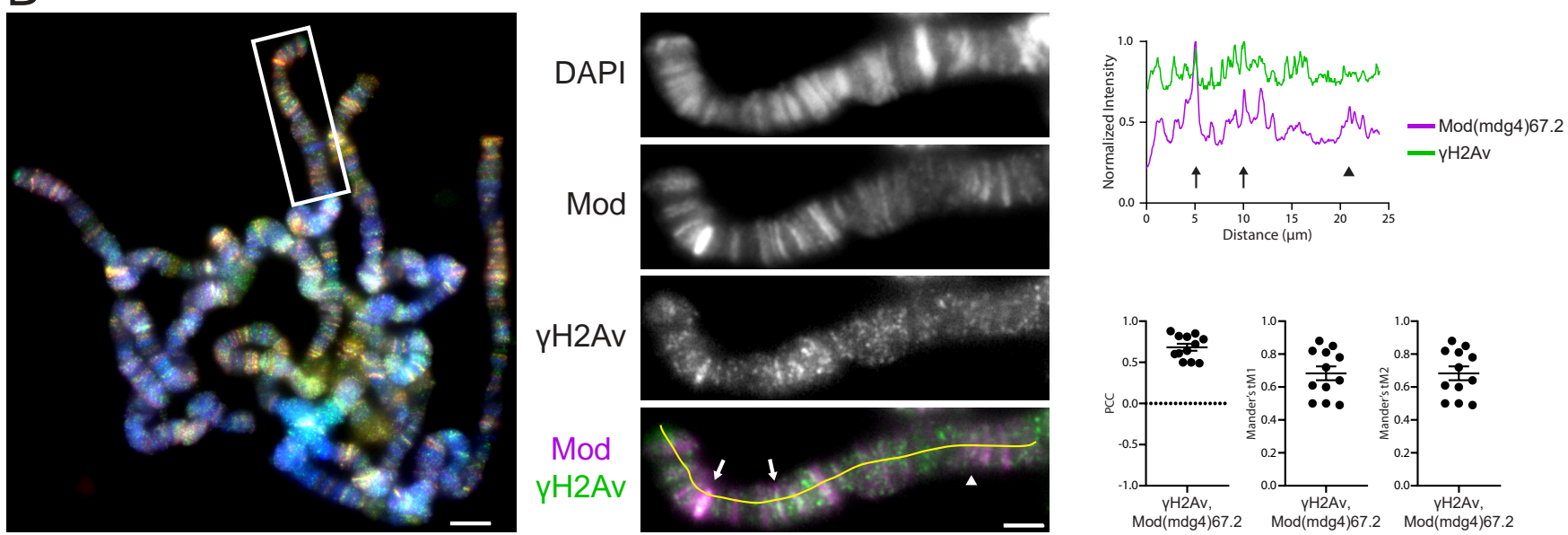

Mod, yH2Av, DAPI

\section{C}

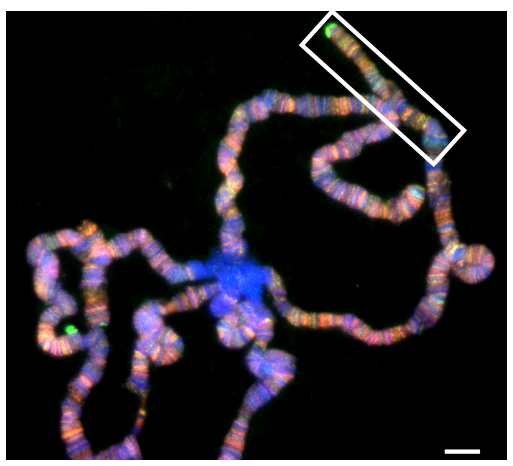

DAPI

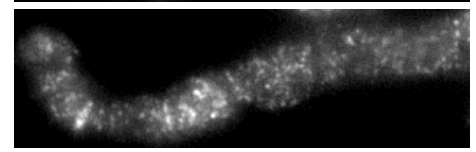

Mod

yH2Av
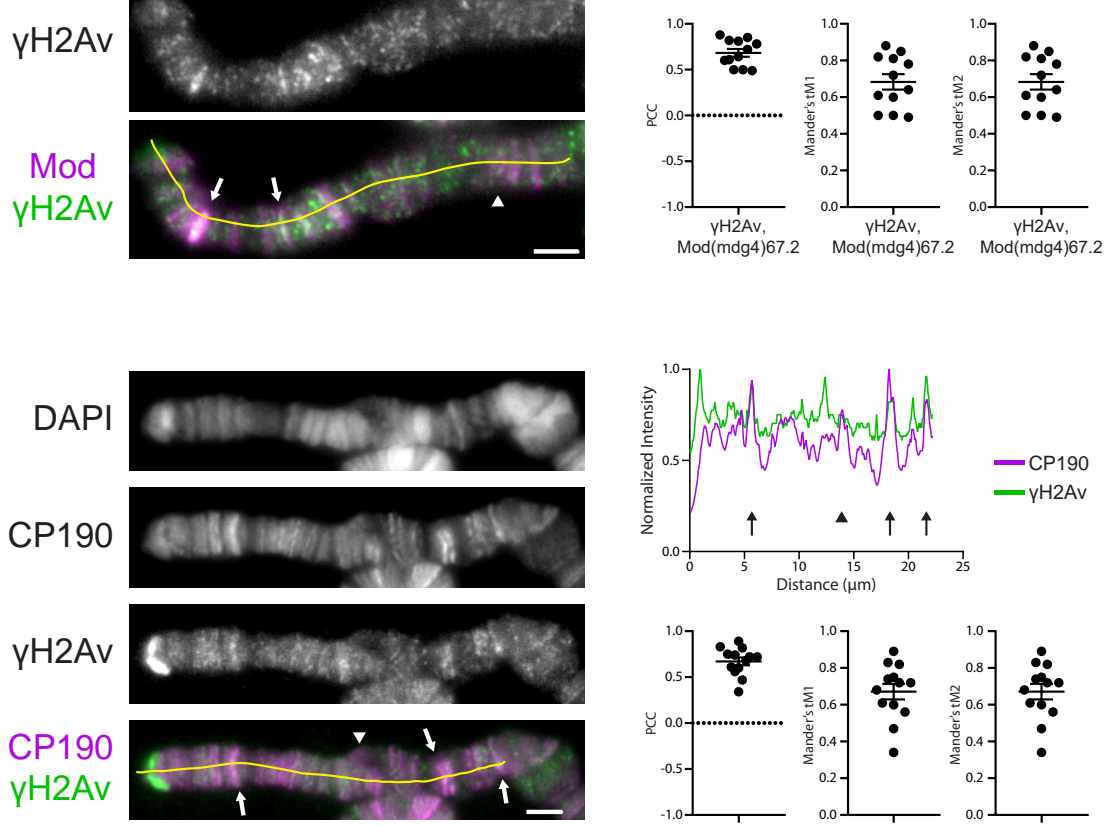

CP190, yH2Av, DAPI

Figure 1. Insulator proteins colocalize with phosphorylated H2Av in Drosophila polytene chromosomes. A. Colocalization of $\gamma \mathrm{H} 2 \mathrm{Av}$ with $\mathrm{Su}(\mathrm{Hw})$. B. Colocalization of $\mathrm{\gamma H} 2 \mathrm{Av}$ with $\mathrm{Mod}(\mathrm{mdg} 4) 67.2$. C. Colocalization of $\mathrm{yH} 2 \mathrm{Av}$ with $\mathrm{CP} 190$. Immunofluorescent micrographs of polytene chromosome squashes obtained from wandering third-instar larvae are shown on the left. Magnified insets are shown in the middle, corresponding to the white boxes in the figures on the left. Scale bars are $5 \mu \mathrm{m}$ in the figures and $2 \mu \mathrm{m}$ in the insets. Insets are shown as RGB merge, with DAPI on the blue channel, $\gamma \mathrm{H} 2 \mathrm{Av}$ on the green channel, and various insulator proteins on the red channel. Red and green channels are shown independently in grey scale and merged as magenta and green. On the right are linescans performed in FIJI (Schindelin et al., 2012), corresponding to the yellow lines in the merged insets. Linescan intensities were normalized by dividing each value by the maximum intensity recorded on each channel. Arrows in the images and respective linescans denote regions of strong colocalization while arrowheads denote regions enriched for insulator proteins but not $\mathrm{\gamma H} 2 \mathrm{Av}$. Pearson's Correlation Coefficient (PCC) for $\gamma \mathrm{H} 2 \mathrm{Av}$ signal with each insulator protein signal is plotted, with each point representing the polytene genome of each cell. Similarly, Mander's tM1 (the proportion of $\gamma$ H2Av-positive pixels that also contain signal from antibodies against insulator proteins) and tM2 (the proportion of insulator protein-positive pixels that also contain signal from the $\mathrm{\gamma H} 2 \mathrm{Av}$ antibody) are plotted in each cell. Error bars represent one standard error of the mean. 
bioRxiv preprint doi: https://doi.org/10.1101/2021.02.23.432395; this version posted February 23, 2021. The copyright holder for this preprint (which was not certified by peer review) is the author/funder. All rights reserved. No reuse allowed without permission.
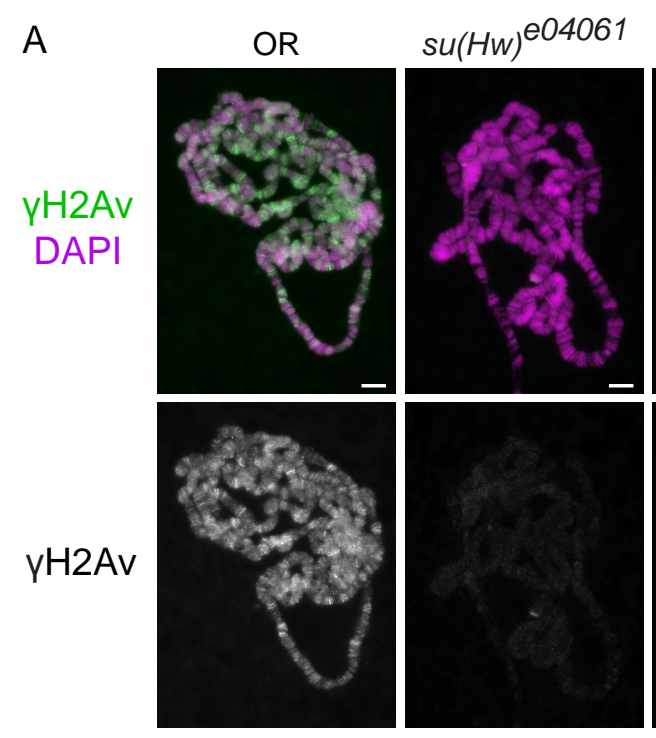
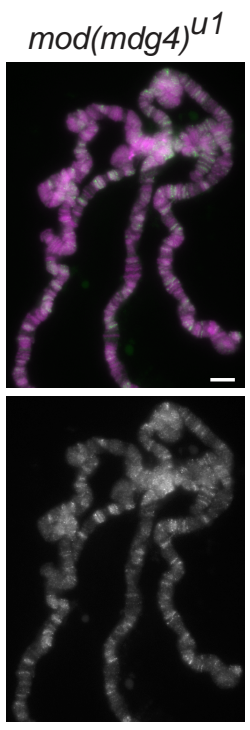
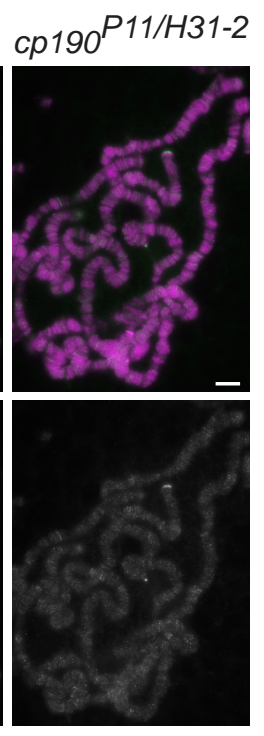

B

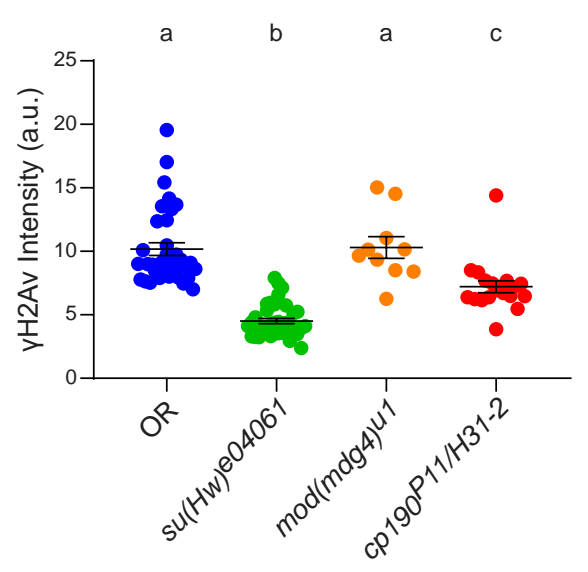

Figure 2. Phosphorylation of H2Av in polytene chromosomes is inhibited in insulator protein mutants. A. Immunostaining of polytene chromosomes from wandering third instar Drosophila larval salivary glands from various insulator mutant genotypes (listed above each figure). An antibody against phosphorylated $\mathrm{H} 2 \mathrm{Av}(\mathrm{\gamma H} 2 \mathrm{Av})$ was used (green in the merged image, shown alone in greyscale below). Scale bars represent $5 \mu \mathrm{m}$. B. Quantification of fluorescent signals from immunostains with $\mathrm{\gamma H} 2 \mathrm{Av}$ shown in A. Each point represents the polytene genome of an individual cell. Error bars represent one standard error of the mean. Letters above the data indicate statistical groupings as determined using an ANOVA performed with Games-Howell's multiple corrections. 
Two values are reported, one for each channel, with values ranging from 0 (representing no spatial overlap between signals) and 1 (representing complete overlap of signals).

An extensive genome-wide association between $\mathrm{\gamma H} 2 \mathrm{Av}$ and $\mathrm{Su}(\mathrm{Hw})$ is illustrated by the positive PCC values and MOC values above 0.5 . Given the fundamental role of $\gamma \mathrm{H} 2 \mathrm{Av}$ in DNA repair, the tight association of $\mathrm{Su}(\mathrm{Hw})$ with phosphorylated H2Av supports the notion that $\mathrm{Su}(\mathrm{Hw})$ is involved in maintaining genome integrity (Lankenau et al., 2000, Hsu et al., 2020). Immunostaining experiments yield similar results for $\operatorname{Mod}(\operatorname{mdg} 4) 67.2$, the isoform of the $\bmod (m d g 4)$ locus associated with gypsy insulator function (Figure 1B). Bands of $\operatorname{Mod}(\operatorname{mdg} 4) 67.2$ are seen to overlap with bands of $\gamma \mathrm{H} 2 \mathrm{Av}$ (Figure $1 \mathrm{~B}$ ) and linescans reveal a close covariance (Figure 1B). The same pattern is recapitulated when examining images and linescans of CP190 (Figure 1C), an insulator protein found at gypsy and CTCF insulator sites (Pai et al., 2004, Mohan et al., 2007). Quantitative analysis of signal colocalization shows strong positive correlation values when examining genome-wide signal in polytene chromosomes for both $\operatorname{Mod}(\operatorname{mdg} 4)$ and CP190 with $\gamma \mathrm{H} 2 \mathrm{Av}$ (Figure $1 \mathrm{~B}, \mathrm{C}$ ). These findings suggest that $\mathrm{\gamma H} 2 \mathrm{Av}$ colocalizes with gypsy insulator proteins throughout the genome under normal developmental conditions.

\section{Phosphorylated H2Av Is Stabilized by gypsy Insulator Components}

To further our understanding of the relationship between $\mathrm{\gamma H} 2 \mathrm{Av}$ and insulator complexes we asked whether or not mutation of genes coding for gypsy insulator complex members would affect $\mathrm{H} 2 \mathrm{Av}$ phosphorylation. Immunostaining of polytene chromatin revealed an almost complete elimination of $\mathrm{\gamma H} 2 \mathrm{Av}$ in the chromatin of $s u(H w)^{004061}$ mutants (Figure 2). Mutation of $c p 190$ also resulted in less $\mathrm{YH} 2 \mathrm{Av}$ signal in the immunostained chromosomes, however, the reduction was not as severe as was seen in the $s u(H w)^{e 04061}$ mutant (Figure 2). In contrast with $s u(H w)$ and $c p 190$, mutation of $\bmod (\operatorname{mdg} 4)$ had no significant effect on the amount of H2Av phosphorylation in chromatin (Figure 2). These results suggest that $\mathrm{Su}(\mathrm{Hw})$ and $\mathrm{CP} 190$, but not $\mathrm{Mod}(\mathrm{mdg} 4) 67.2$, are necessary for sustaining $\mathrm{H} 2 \mathrm{Av}$ phosphorylation and may hint at a mechanism that depends on specific interactions between $\mathrm{H} 2 \mathrm{Av}$ with some but not all gypsy insulator components.

As interactions between insulator proteins are generally required for canonical insulator functions (Geyer and Corces, 1992, Bonchuk et al., 2015, Golovnin et al., 2016, Melnikova, Kostyuchenko, Parshikov, et al., 2018, Melnikova et al., 2019), we examined the relationship between each of these insulator proteins and $\mathrm{\gamma H} 2 \mathrm{Av}$ in various insulator mutant backgrounds. Notably, the colocalization between $\mathrm{Mod}(\mathrm{mdg} 4) 67.2$ and $\mathrm{\gamma H} 2 \mathrm{Av}$ was strongly reduced in the $s u(H w)^{e 04061}$ background (Figure 3A, F). This is not surprising given both that $\mathrm{\gamma H} 2 \mathrm{Av}$ signal is significantly reduced in $\mathrm{su}(\mathrm{Hw})^{\text {e04061 }}$ (Figure 2) and that Mod(mdg4)67.2 does not bind at $\mathrm{Su}(\mathrm{Hw})$ sites in the absence of $\mathrm{Su}(\mathrm{Hw})$ (Ghosh et al., 2001). Linescans of polytene chromosomes and quantitative colocalization analysis show no correlation between the signals from Mod(mdg4)67.2 and $\mathrm{\gamma H}_{2} \mathrm{Av}$ in $\mathrm{su}(\mathrm{Hw})^{e 04061}$ mutant polytene chromosomes (Figure 3A).
Similarly, immunostaining for $\mathrm{Su}(\mathrm{Hw})$ in the loss of function $\bmod (\operatorname{mdg} 4)^{u 1}$ mutant revealed significantly less colocalization as seen by visual inspection, linescans, and quantitative analysis (Figure 3B, F). Linescans from immunostains of the $\bmod (\operatorname{mdg} 4)^{u 1}$ mutant with either $\mathrm{Su}(\mathrm{Hw}$ ) (Figure 3C) or CP190 (Figure 3D) along with $\mathrm{\gamma H} 2 \mathrm{Av}$ demonstrate a further disruption of the interaction between $\mathrm{\gamma H} 2 \mathrm{Av}$ and insulator complexes. The lack of colocalization between $\mathrm{\gamma H} 2 \mathrm{Av}$ and $\mathrm{Su}(\mathrm{Hw})$ or $\mathrm{CP} 190$ is reflected in decreased PCC values (Figure 3F), with negative values for CP190 indicating anticorrelation between the $\mathrm{CP} 190$ and $\gamma \mathrm{H} 2 \mathrm{Av}$ signals. $\mathrm{Su}(\mathrm{Hw})$ was also found to colocalize less with $\mathrm{\gamma H} 2 \mathrm{Av}$ in the null $c p 190^{\text {P11-H31-2 }}$ mutant background through the area of chromosome $2 \mathrm{R}$ examined (Figure 3E) and genome wide (Figure 3F). Taken together, these results indicate that stable accumulation of $\mathrm{HH}_{2} \mathrm{Av}$ in $\mathrm{Su}(\mathrm{Hw})$ insulator sites requires having the entire insulator complex intact. It remains unclear which $\mathrm{Su}(\mathrm{Hw})$ insulator protein serves as the binding partner of $\mathrm{\gamma H} 2 \mathrm{Av}$, or if this colocalization requires that $\mathrm{\gamma H}_{2} \mathrm{Av}$ interact with more than one insulator protein or other unknown protein.

\section{H2Av Is Phosphorylated in Insulator Bodies}

Upon our finding that $\mathrm{pH} 2 \mathrm{Av}$ colocalizes with insulator proteins in the genome in an insulator-dependent manner, we next asked whether or not this interaction was maintained once insulator proteins aggregate into bodies under osmotic stress conditions. Insulator bodies represent a special case for insulator activity: proteins bound to insulator sites in the genome can leave chromatin and associate to form insulator bodies (Gerasimova and Corces, 1998). The exact purpose of these bodies remains unknown, but recent reports have shed new light on their formation and dynamics. In a previous work, our lab demonstrated that insulator bodies form after dissociation of insulator proteins from chromatin and that insulator body formation can be induced under conditions of high osmotic pressure (Schoborg et al., 2013).

Immunostaining of S2 cells for $\mathrm{\gamma H} 2 \mathrm{Av}$ and $\mathrm{Su}(\mathrm{Hw})$ in isotonic cell culture media shows no visibly apparent pattern between the two proteins (Figure 4A). Linescans support this point, with little covariance seen between the signals (Figure 4A). Incubation of S2 cells in hypertonic media (containing an additional $250 \mathrm{mM} \mathrm{NaCl}$ ) induced strong insulator body formation as previously reported (Schoborg et al., 2013). Immunostaining shows clear localization of $\mathrm{\gamma H} 2 \mathrm{Av}$ to insulator bodies (Figure 4B). Linescans drawn through the insulator bodies confirm this finding, with each signal following the same pattern (Figure 4B). The vast majority of observed insulator bodies formed during osmotic stress were positive for $\mathrm{yH} 2 \mathrm{Av}$ (92.1\%, Figure 4C). A number of smaller insulator bodies containing $\mathrm{\gamma H} 2 \mathrm{Av}$ also formed under isotonic conditions, implying that some amount of phosphorylated $\mathrm{H} 2 \mathrm{Av}$ may be involved in insulator body function during normal cellular conditions. Alternatively, these cells may represent cells undergoing apoptosis (Schoborg et al., 2013). Quantitative colocalization analysis shows a clear and significant increase in colocalization between $\mathrm{Su}(\mathrm{Hw})$ and $\mathrm{YH} 2 \mathrm{Av}$ after hypertonic stress, whereas there is no colocalization between the two signals in isotonic media (Figure 4D). 


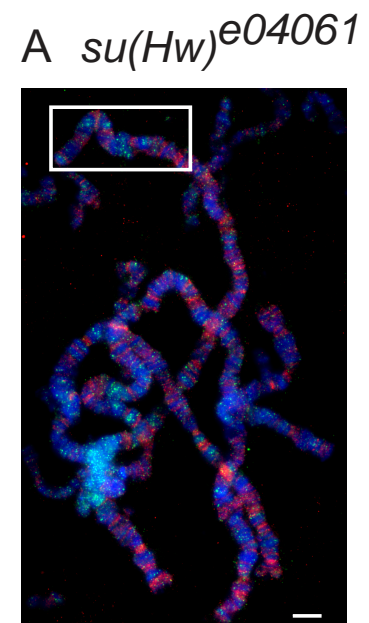

Mod, $\mathrm{yH} 2 \mathrm{Av}$, DAPI
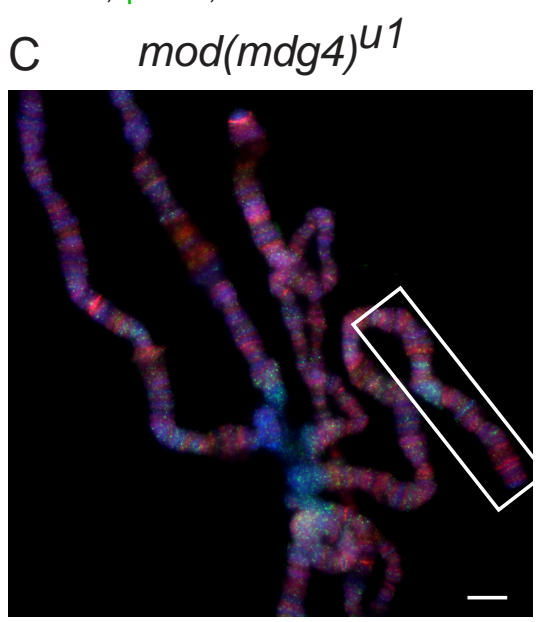

$\mathrm{Su}(\mathrm{Hw}), \mathrm{yH} 2 \mathrm{Av}, \mathrm{DAPI}$

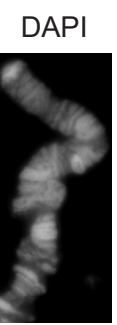

Mod
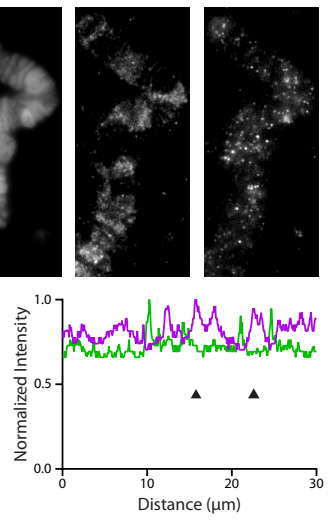

— $\operatorname{Mod}(\mathrm{mdg} 4) 67.2-\mathrm{\gamma H} 2 \mathrm{Av}$
Mod
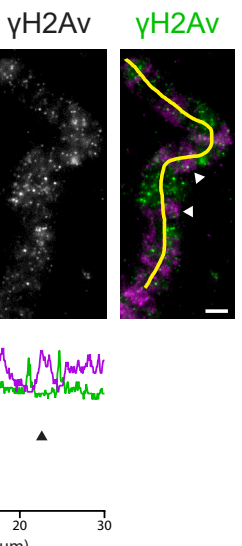

B $\operatorname{su}(H w)^{e 04061}$

DAPI Su(Hw)yH2Avy
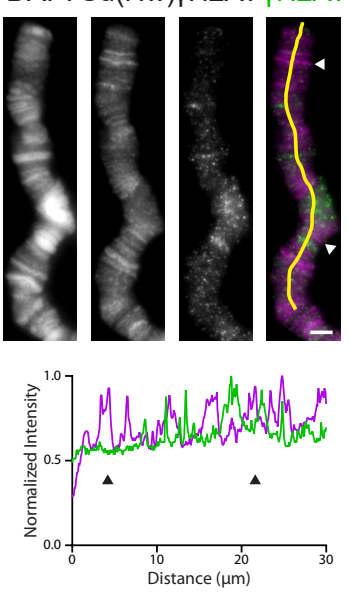

$-\mathrm{Su}(\mathrm{Hw})-\mathrm{vH}_{2 \mathrm{Av}}$

$\mathrm{Su}(\mathrm{Hw})$

DAPI $\mathrm{Su}(\mathrm{Hw})$ yH2Av $y \mathrm{H} 2 \mathrm{Av}$
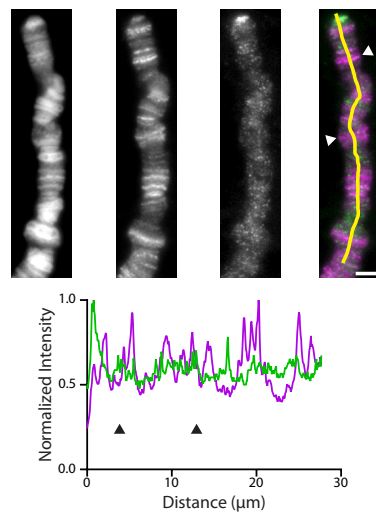

$-\mathrm{Su}(\mathrm{Hw}) \quad-\mathrm{rH} 2 \mathrm{Av}$
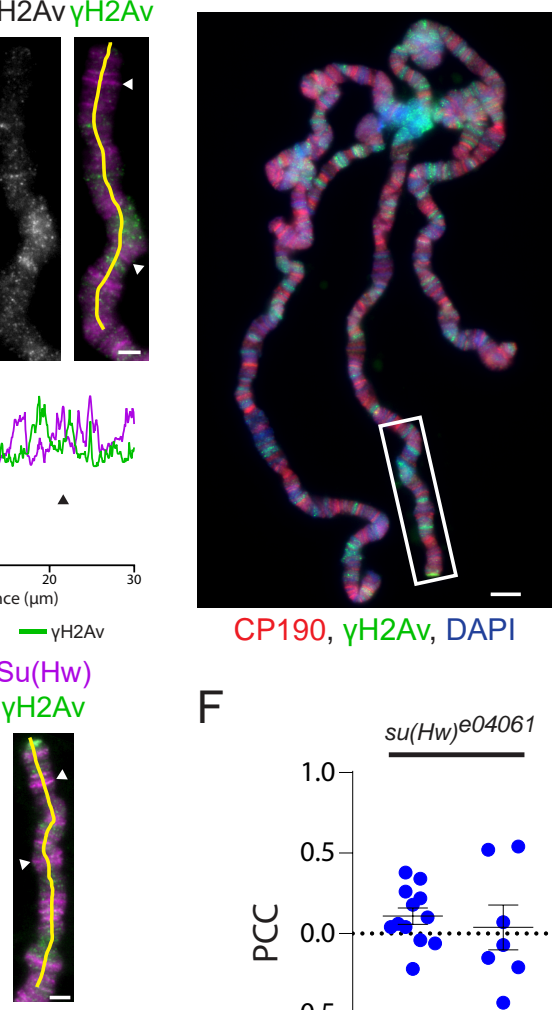

CP190, yH2Av, DAPI

F

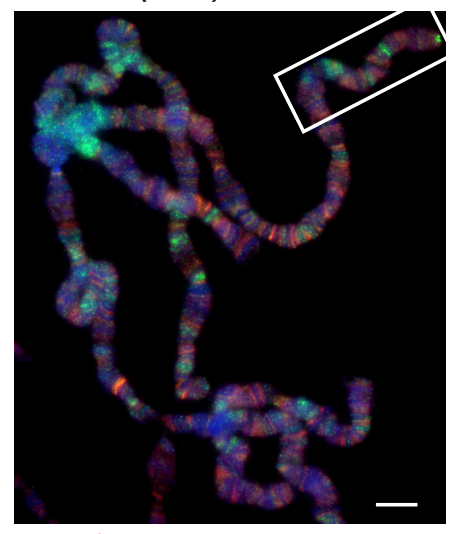

CP190, yH2Av, DAPI

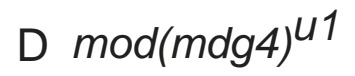

CP190

DAPI CP190 yH2Av $\mathrm{yH} 2 \mathrm{Av}$
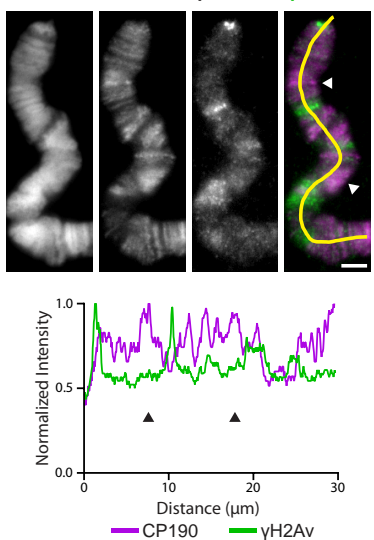

CP190

DAPI CP190 yH2Av $y \mathrm{H} 2 \mathrm{Av}$
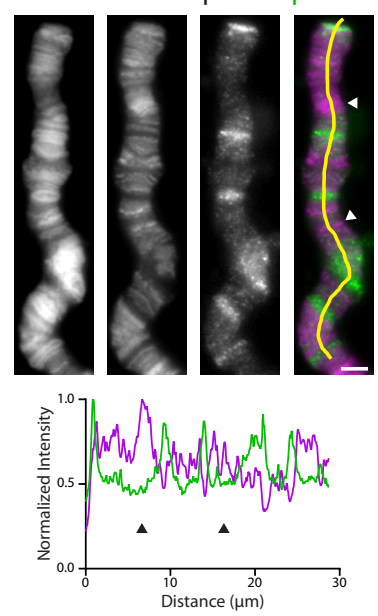

- $\mathrm{CP} 190-\mathrm{rH} 2 \mathrm{Av}$

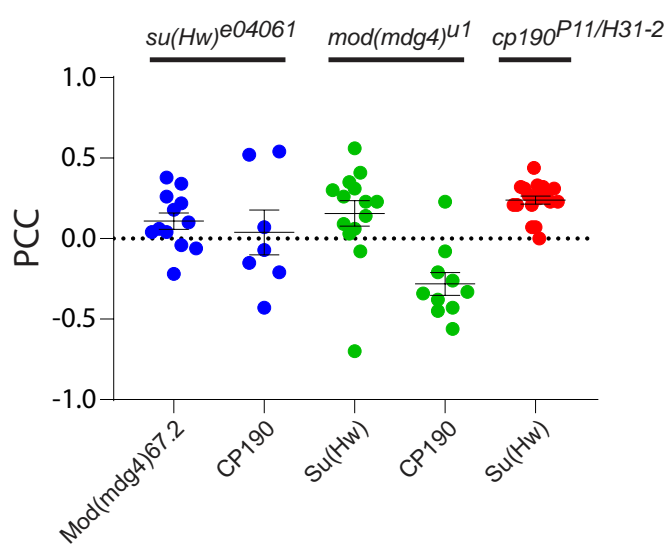

$\mathrm{Su}(\mathrm{Hw}), \mathrm{yH} 2 \mathrm{Av}, \mathrm{DAPI}$

Figure 3. Insulator components are interdependent for their colocalization with $\mathbf{\gamma H 2 A v}$ in Drosophila polytene chromosomes. A. Colocalization of $\mathrm{yH} 2 \mathrm{Av}$ with Mod(mdg4)67.2 in su(Hw)e04061. B. Colocalization of $\gamma \mathrm{H} 2 \mathrm{Av}$ with $\mathrm{CP} 190 \mathrm{in} s u(\mathrm{Hw})^{e 04061}$. C. Colocalization of $\mathrm{\gamma H} 2 \mathrm{Av}$ with Su(Hw) in mod(mdg4)u1. D. Colocalization of $\mathrm{\gamma H} 2 \mathrm{Av}$ with $\mathrm{CP} 190 \mathrm{in} \bmod (m d g 4)^{u 1}$. E. Colocalization of $\mathrm{\gamma H} 2 \mathrm{Av}$ with $\mathrm{Su}(\mathrm{Hw})$ in $c p 190^{\mathrm{P} 11 / \mathrm{H} 31-2}$. F. Pearson's Correlation Coefficient (PCC) for $\mathrm{\gamma H} 2 \mathrm{Av}$ signal with each insulator signal is plotted, with each point representing the polytene genome of each cell. Error bars represent one standard error of the mean. PCC values are grouped by genotype $\left(\mathrm{red}=s u(H w)^{e 04061}\right.$, green $=\bmod (\operatorname{mdg} 4)^{u 1}$, blue $\left.=c p 190^{p 11 / H 31-2}\right)$. Immunofluorescent micrographs of polytene chromosome squashes obtained from wandering third-instar larvae are shown on the left. Magnified insets are shown to the right of each figure, corresponding to the white boxes in the figures on the left. Scale bars are $5 \mu \mathrm{m}$ in the figures and $2 \mu \mathrm{m}$ in the insets. Insets are shown as RGB merge, with DAPI on the blue channel, $\gamma \mathrm{H} 2 \mathrm{Av}$ on the green channel, and various insulator proteins on the red channel. Red and green channels are shown independently in grey scale and merged as magenta and green. Beneath the insets are linescans performed in FIJI (Schindelin et al., 2012), corresponding to the yellow lines in the merged insets. Linescan intensities were normalized by dividing each value by the maximum intensity recorded on each channel. Arrows in the images and respective linescans denote regions of strong colocalization while arrowheads denote regions enriched for insulator proteins but not $\mathrm{\gamma H} 2 \mathrm{Av}$. 
A

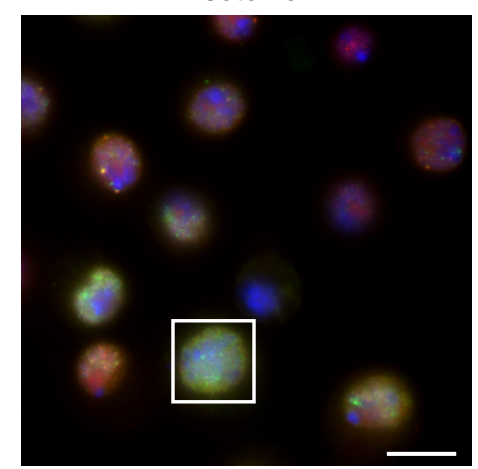

$\mathrm{Su}(\mathrm{Hw}), \mathrm{\gamma H} 2 \mathrm{Av}, \mathrm{DAPI}$
Hypertonic

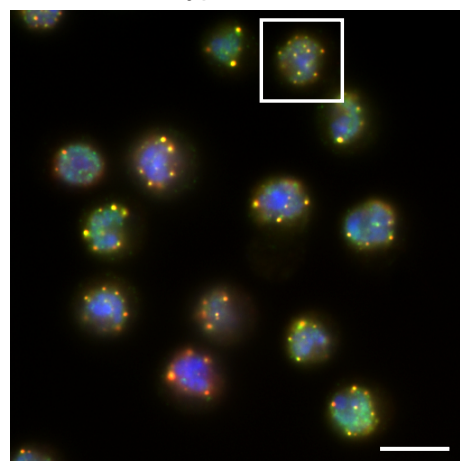

$\mathrm{Su}(\mathrm{Hw}), \mathrm{yH} 2 \mathrm{Av}, \mathrm{DAPI}$
B
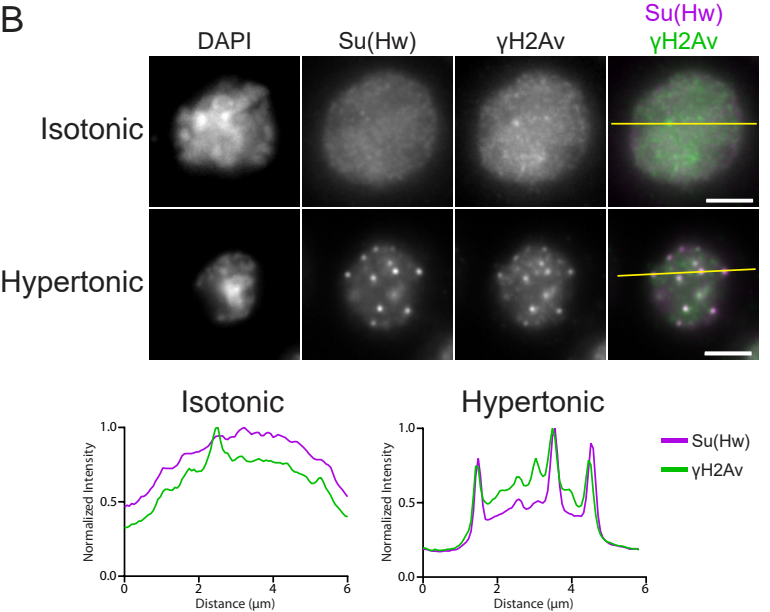

C

\begin{tabular}{|c|c|c|}
\hline Media & \# of insulator bodies & \% of bodies with $\mathrm{\gamma H2Av}$ \\
\hline Isotonic & 95 & 55.8 \\
\hline Hypertonic & 850 & 92.1 \\
\hline
\end{tabular}

D

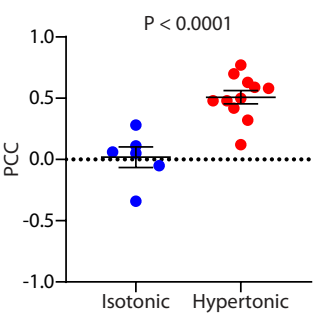

F

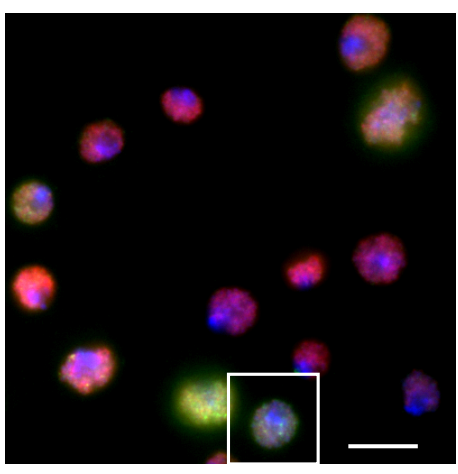

H2Av, yH2Av, DAPI
E Hypertonic

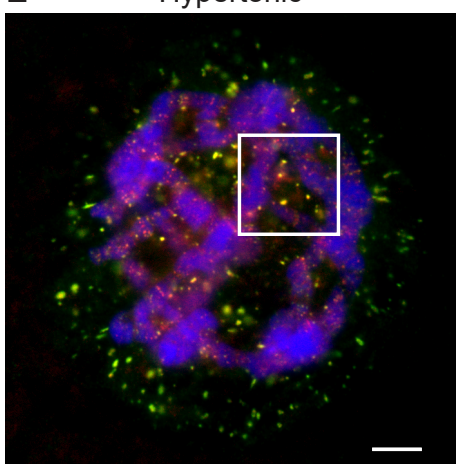

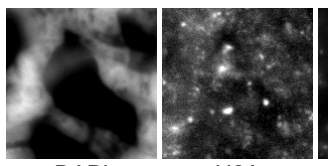

DAPI
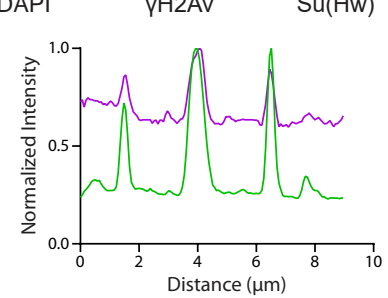

G

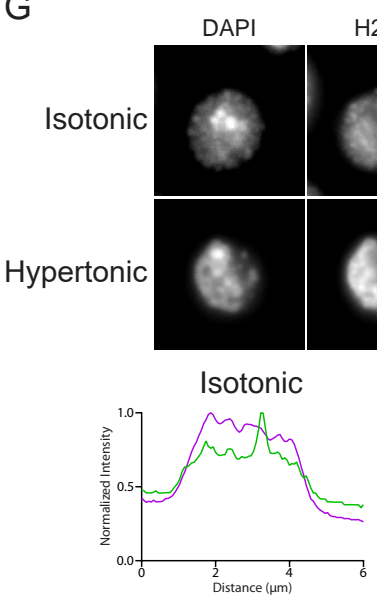

a yH2Av

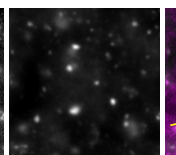

$\mathrm{Su}(\mathrm{Hw})$

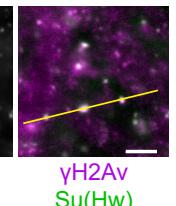

$-\mathrm{vH} 2 \mathrm{Av}$
Hypertonic

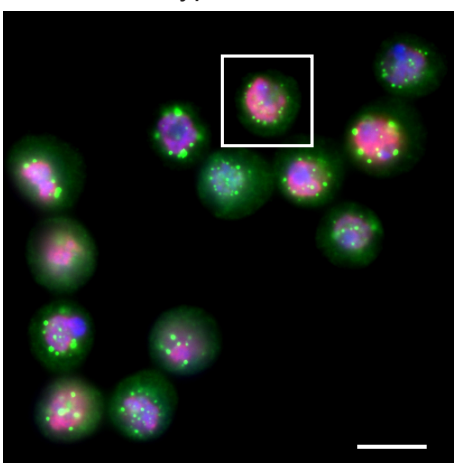

H2Av, yH2Av, DAPI
$\mathrm{H} 2 \mathrm{Av}$ pH2Av

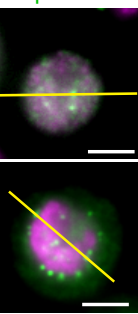

Hypertonic

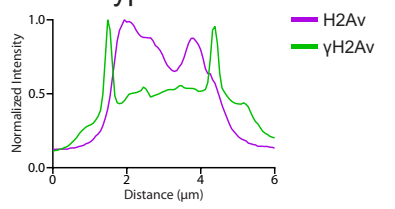

Figure 4. Phosphorylated H2Av is a component of insulator bodies. A. Immunostaining of Drosophila S2 cells in isotonic media (left) and hypertonic media (right). Insulator bodies formed during osmotic stress are labelled with $\mathrm{Su}(\mathrm{Hw})$ (red). Phosphorylated H2Av (green) colocalizes with Su(Hw) in insulator bodies. Scale bars are $5 \mu \mathrm{m}$ and insets for analysis are delineated by white boxes. B. Magnified view of insets showing insulator body formation in hypertonic, but not isotonic, conditions. Insets are shown as RGB merge, with DAPI on the blue channel, $\mathrm{yH} 2 \mathrm{Av}$ on the green channel, and Su(Hw) on the red channel. Red and green channels are shown independently in grey scale and merged as magenta and green. Beneath the insets are linescans corresponding to the yellow lines in the merged insets. Scale bars represent $2 \mu \mathrm{m}$. C. Table showing the ratio of insulator bodies with significant amounts of $\mathrm{\gamma H} 2 \mathrm{Av}$. D. Pearson's Correlation Coefficient (PCC) for $\mathrm{\gamma H} 2 \mathrm{Av}$ signal with $\mathrm{Su}(\mathrm{Hw})$ signal is plotted for isotonic versus hypertonic conditions, with each point representing a field of S2 cells. Error bars represent one standard error of the mean. The P-value was determined using an unpaired two-tailed Student's T-test. E. Polytene chromosomes from wandering third instar larval salivary glands under hypertonic conditions. Colocalization between $\mathrm{\gamma H} 2 \mathrm{Av}$ and $\mathrm{Su}(\mathrm{Hw})$ is shown in the insets and linescan. The scale bar in the wide view represents $5 \mu \mathrm{m}$, the scale bar in the inset represents $2 \mu \mathrm{m}$. F. Immunostaining of Drosophila S2 cells in isotonic media (left) and hypertonic media (right). Phosphorylated H2Av (green) localizes to insulator bodies while unphosphorylated H2Av (red) does not. Scale bars are $5 \mu \mathrm{m}$ and insets for analysis are delineated by white boxes. G. Magnified insets from F. Beneath the insets are linescans corresponding to the yellow lines in the merged insets. Scale bars represent $2 \mu \mathrm{m}$. Linescan intensities in B, E, and $\mathrm{G}$ were normalized by dividing each value by the maximum intensity recorded on each channel. 
Insulator bodies have previously been shown to form in other cell types such as the polytene cells of the larval salivary gland (Schoborg et al., 2013). In order to determine whether or not phosphorylated H2Av was present in insulator bodies formed under osmotic stress conditions, larval salivary glands were treated in hypertonic solution and immunostained for $\mathrm{Su}(\mathrm{Hw})$ and $\mathrm{yH} 2 \mathrm{Av}$. Analysis of the micrographs reveals strong colocalization of the $\mathrm{YH} 2 \mathrm{Av}$ antibody in the insulator bodies labelled by $\mathrm{Su}(\mathrm{Hw})$ in salivary gland cells (Figure $4 \mathrm{E}$ ). There is no significant accumulation of unphosphorylated $\mathrm{H} 2 \mathrm{Av}$ in these insulator bodies (Figure $4 \mathrm{~F}$ and $4 G)$, suggesting that phosphorylation of this histone variant is important for its localization to insulator bodies. The mechanism behind how insulators leave chromatin and form bodies is unknown, and the involvement of $\gamma \mathrm{H} 2 \mathrm{Av}$ adds an unexpected layer to this question. The presence of $\mathrm{\gamma H} 2 \mathrm{Av}$ in insulator bodies may also have implications in how cells respond to DNA damage.

\section{Phosphatase Inhibition Affects Interactions between $\mathrm{YH} 2 \mathrm{Av}$ and gypsy Insulators}

$\mathrm{H} 2 \mathrm{Av}$ phosphorylation is tightly regulated by a network of kinases and phosphatases that integrate signals from various aspects of cellular activity including DNA damage repair (Sirbu and Cortez, 2013). Dephosphorylation of mammalian $\gamma \mathrm{H} 2 \mathrm{AX}$ is mitigated primarily through Protein Phosphatase 2A (PP2A), but also occurs through the activity of other phosphatases, including Protein Phosphatase 4 (PP4) (Nakada et al., 2008) and Wip1 phosphatase (Macurek et al., 2010). In order to determine how H2Av phosphorylation affects genome dynamics, we used okadaic acid (OA), a potent inhibitor of serine/threonine phosphatases PP1 and PP2A in vitro and in vivo (Bialojan and Takai, 1988, Haystead et al., 1989). As okadaic acid may affect other phosphatases at high concentrations (Honkanen and Golden, 2002), a concentration of $50 \mathrm{nM}$ was chosen. Third-instar larval salivary glands were dissected then incubated in okadaic acid before fixation, squashing, and immunostaining. Examination of the polytene chromosomes showed no adverse effect of okadaic acid on the binding of insulator proteins or their colocalization with phosphorylated $\mathrm{H} 2 \mathrm{Av}$ (Figure 5A, C, D). Quantification of the antibody signals indicates a significant increase in the amount of $\mathrm{Su}(\mathrm{Hw})$ signal in the presence of okadaic acid compared to untreated samples (Figure 5B). Notably, there is also an increase in the amount of phosphorylated $\mathrm{H} 2 \mathrm{Av}$ bound to the polytene chromatin in the presence of okadaic acid compared to the untreated control (Figure 5B). This would seem to indicate that the okadaic acid is inhibiting PP2A from dephosphorylating $\mathrm{\gamma H} 2 \mathrm{Av}$ in chromatin, resulting in a significant accumulation of the modified histone variant. This is in conflict with findings from human cell culture, which were shown to increase cellular levels of $\mathrm{H} 2 \mathrm{AX}$ phosphorylation in response to DNA damaging agents in the presence of $25 \mathrm{nM}$ okadaic acid, but not in the presence of okadaic acid alone (Chowdhury et al., 2005). The difference may be a result either of the lower concentration of okadaic acid used in the previous study, or of the innate differences between the two model systems, i.e. the dual nature of Drosophila $\mathrm{H} 2 \mathrm{Av}$, which has the combined functions of mammalian $\mathrm{H} 2 \mathrm{AX}$ and H2AZ (van Daal et al., 1988). The increased $\mathrm{HH} 2 \mathrm{Av}$ signal in the presence of okadaic acid and in the absence of exogenous DNA damage may therefore support the H2AZ-like transcriptional regulation role in insulator function.

Intriguingly, immunostaining of salivary glands from insulator mutants after incubation in okadaic acid revealed a significant rescue of both $\mathrm{H} 2 \mathrm{Av}$ phosphorylation and its colocalization with components of the gypsy insulator complex. Close examination of the polytene chromosomes in the $\mathrm{su}(\mathrm{Hw})^{e 04061}$ mutant shows many sites of colocalization between $\mathrm{\gamma H} 2 \mathrm{Av}$ with both $\mathrm{Mod}(\mathrm{mdg} 4) 67.2$ and CP190 (Figure 6 A, B) which were lacking in the untreated mutant (Figure $3 \mathrm{~A}, \mathrm{~B}$ ). This result suggests that in $\mathrm{su}(\mathrm{Hw})$ mutants, Mod(mdg4)67.2 interacts with CP190 and that this interaction is enhanced by $\mathrm{\gamma H} 2 \mathrm{Av}$. Similar results were obtained when staining for $\mathrm{Su}(\mathrm{Hw})$ and $\mathrm{CP} 190$ in the $\bmod (\operatorname{mdg} 4)^{u 1}$ mutant, with both proteins showing colocalization with phosphorylated H2Av after incubation with okadaic acid (Figure $6 \mathrm{C}, \mathrm{D}$ ). As with the su(Hw)e04061 mutant, this is in contrast to the untreated mutant samples in which $\mathrm{\gamma H} 2 \mathrm{Av}$ and gypsy insulator complexes do not colocalize. Staining of the trans-heterozygous $c p 190^{\text {P11/H31-2 }}$ mutant for $\mathrm{Su}(\mathrm{Hw})$ after okadaic acid treatment yielded the same response, with colocalization between $\mathrm{Su}(\mathrm{Hw})$ and $\mathrm{yH} 2 \mathrm{Av}$ being rescued compared to the untreated mutant (Figure 6E). Colocalization analysis over the entire area of the polytene chromosomes showed strong correlation between the $\mathrm{\gamma H} 2 \mathrm{Av}$ signal and signals from insulator proteins in the okadaic acidtreated insulator mutants (Figure 6F). This is reflected in positive values for Pearson's Correlation Coefficient (PCC), indicating significant correlations between $\mathrm{\gamma H} 2 \mathrm{Av}$ and gypsy insulator signals. This is in contrast to untreated mutant samples, which show a lack of correlation between $\mathrm{\gamma H} 2 \mathrm{Av}$ and gypsy insulator proteins (Figure $3 F)$. These findings point to a model in which $\mathrm{\gamma H} 2 \mathrm{Av}$ acts as a component of gypsy insulators. Phosphorylation of H2Av may be required for gypsy insulator complex formation or stabilization, and may play an essential role in insulator functions.

We next asked if this role of $\mathrm{pH} 2 \mathrm{Av}$ in gypsy insulator dynamics is limited to chromatin-bound insulators or if it extends to other cellular functions of insulators. To this end, S2 cells were exposed to osmotic stress to induce insulator body formation with the goal of determining if $\mathrm{H} 2 \mathrm{Av}$ phosphorylation is required for insulator body formation or recovery after stress (Figure 7A). As a control to ensure okadaic acid alone does not induce body formation, cells were incubated in isotonic media with okadaic acid. These cells showed similarly low numbers of insulator bodies per cell as cells in untreated isotonic media (Figure 7B). Osmotic stress was introduced by increasing the salt concentration (Schoborg et al., 2013), resulting in the formation of many insulator bodies (Figure $7 A, C)$. No significant difference was seen in the ratio of cells that contained insulator bodies when comparing okadaic acid-treated and -untreated samples (Figure 7B). Of particular interest, however, is the finding that after the osmotic stress media is replaced with isotonic media, cells treated with okadaic acid recover significantly less than cells not exposed to okadaic acid, retaining a greater number of insulator bodies throughout recovery (Figure 7B, C). These results put into context our finding that $\mathrm{\gamma H} 2 \mathrm{Av}$ is present in insulator bodies (Figure 3) and imply that phosphorylated H2Av must be maintained in the insulator body as part of the normal osmotic response. Preventing dephosphorylation of $\mathrm{\gamma H} 2 \mathrm{Av}$ by phosphatase inhibition prevents 
bioRxiv preprint doi: https://doi.org/10.1101/2021.02.23.432395; this version posted February 23, 2021. The copyright holder for this preprint (which was not certified by peer review) is the author/funder. All rights reserved. No reuse allowed without permission.

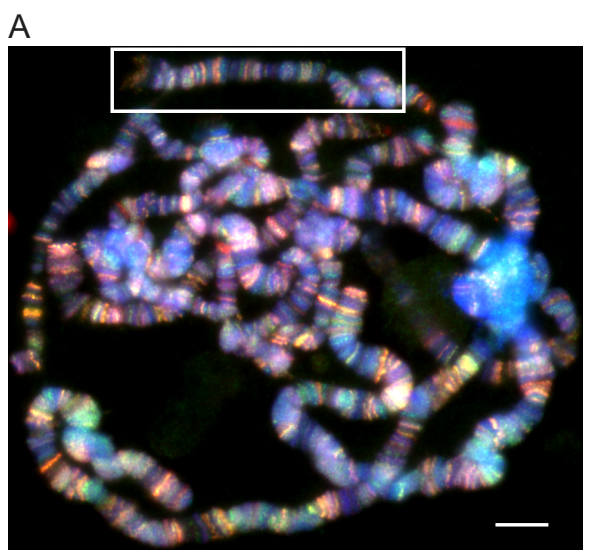

C

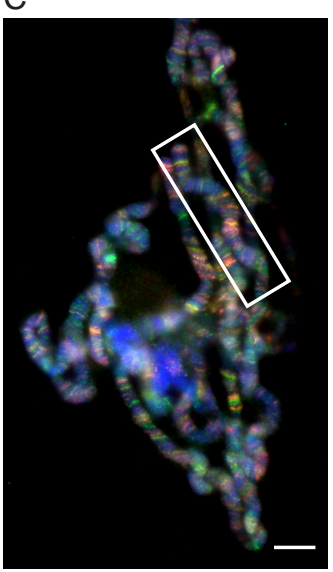

Mod, $\mathrm{yH} 2 \mathrm{Av}$, DAPI

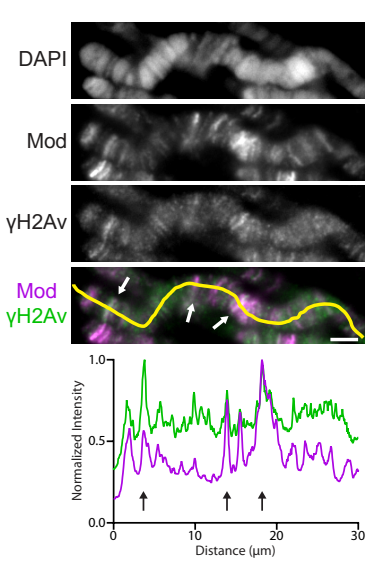

- $\operatorname{Mod}(\mathrm{mdg} 4) 67.2-\mathrm{vH} 2 \mathrm{Av}$

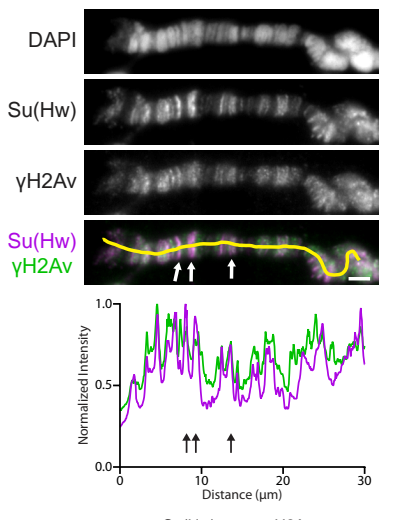

$-\mathrm{Su}(\mathrm{Hw})-\mathrm{rH} 2 \mathrm{Av}$

$\mathrm{D}$
B
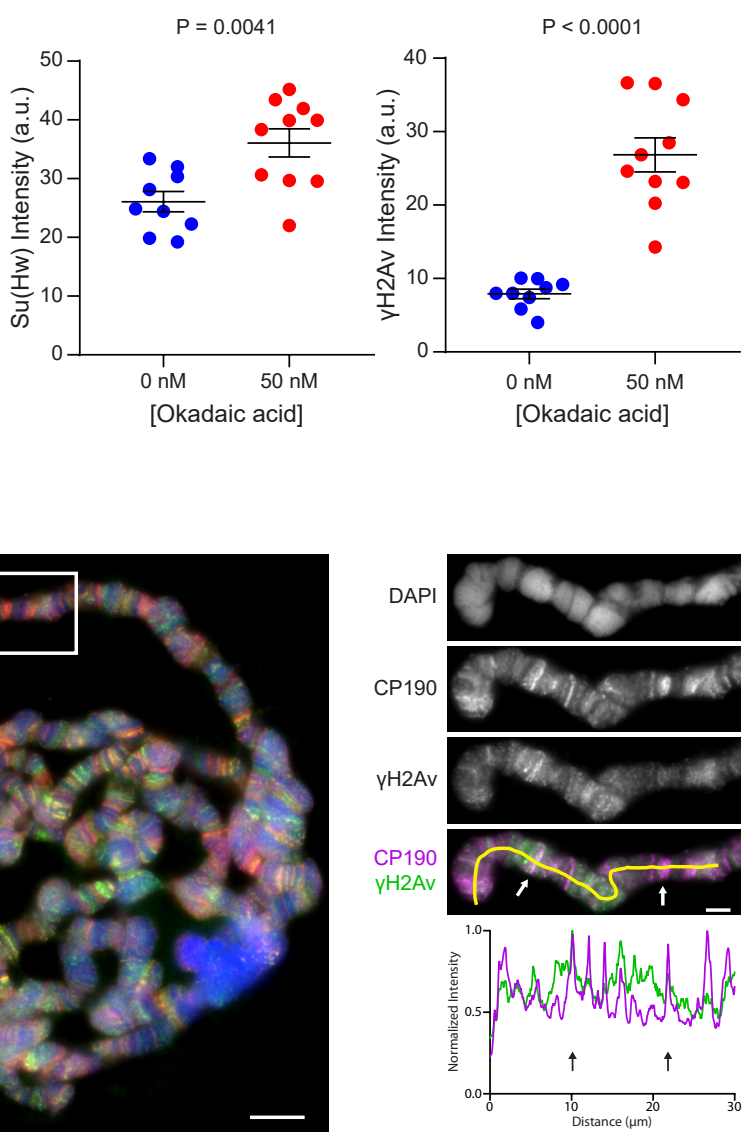

- $\mathrm{CP} 190-\mathrm{rH} 2 \mathrm{Ar}$

Figure 5. Treatment with okadaic acid increases the amount of phosphorylated H2Av on polytene chromosomes. Shown are co-immunostains of polytene chromosomes from salivary glands treated with okadaic acid. A. Immunostaining of $\gamma \mathrm{H} 2 \mathrm{Av}$ with Su(Hw). B. Quantification of the immunostaining data show in A. The intensities of $\mathrm{Su}(\mathrm{Hw})$ and $\mathrm{\gamma H} 2 \mathrm{Av}$ are shown under in the absence and presence of okadaic acid. Each point represents the polytene genome of an individual cell. Error bars represent one standard error of the mean. P-values were determined using unpaired two-tailed Student's T-tests. C. Immunostaining of $\gamma \mathrm{H} 2 \mathrm{Av}$ with $\mathrm{Mod}(\mathrm{mdg} 4) 67.2$. D. Immunostaining of $\mathrm{\gamma H} 2 \mathrm{Av}$ with CP190. Immunofluorescent micrographs of polytene chromosome squashes are shown on the left. Magnified insets are shown to the right of each figure, corresponding to the white boxes in the figures on the left. Scale bars are $5 \mu \mathrm{m}$ in the figures and $2 \mu \mathrm{m}$ in the insets. Insets are shown as RGB merge, with DAPI on the blue channel, $\mathrm{yH} 2 \mathrm{Av}$ on the green channel, and various insulator proteins on the red channel. Red and green channels are shown independently in grey scale and merged as magenta and green. Beneath the insets are linescans corresponding to the yellow lines in the merged insets. Linescan intensities in A, C, and D were normalized by dividing each value by the maximum intensity recorded on each channel. 

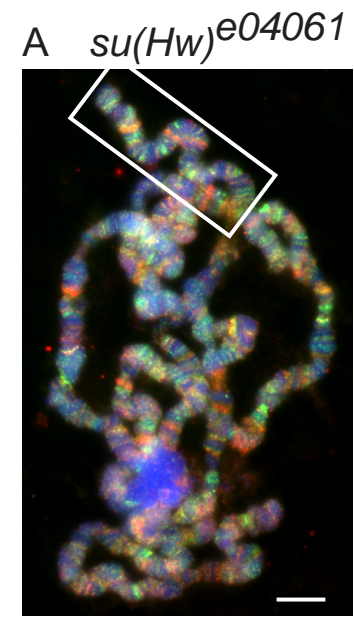

Mod, $\mathrm{yH} 2 \mathrm{Av}$, DAPI

C $\bmod (\operatorname{mdg} 4)^{u 1}$

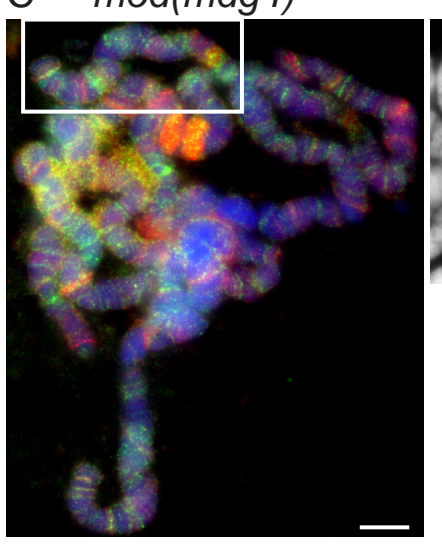

$\mathrm{Su}(\mathrm{Hw}), \mathrm{yH} 2 \mathrm{Av}, \mathrm{DAPI}$
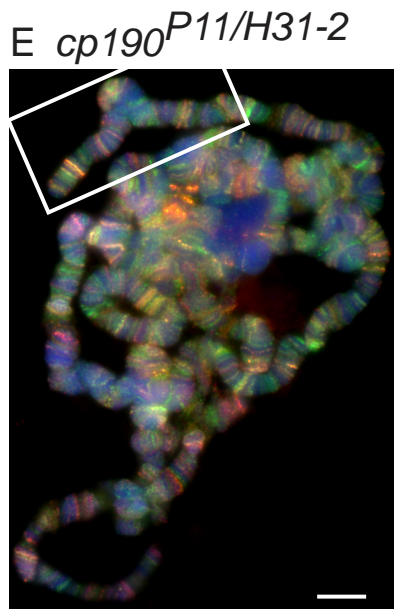

$\mathrm{Su}(\mathrm{Hw}), \mathrm{yH} 2 \mathrm{Av}, \mathrm{DAPI}$
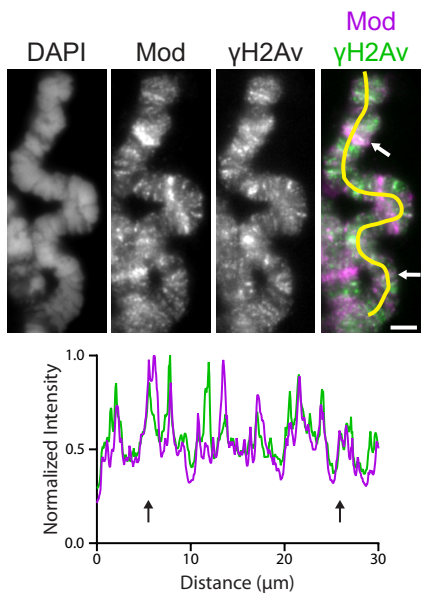

$-\operatorname{Mod}(\operatorname{mdg} 4) 67.2-\mathrm{pH} 2 \mathrm{Av}$
B $\quad \mathrm{su}(\mathrm{Hw})^{\mathrm{e} 04061}$

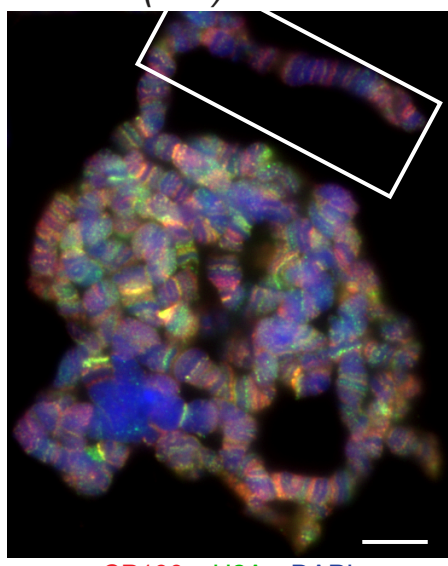

CP190, yH2Av, DAPI

D $\bmod (\operatorname{mog} 4)^{u 1}$

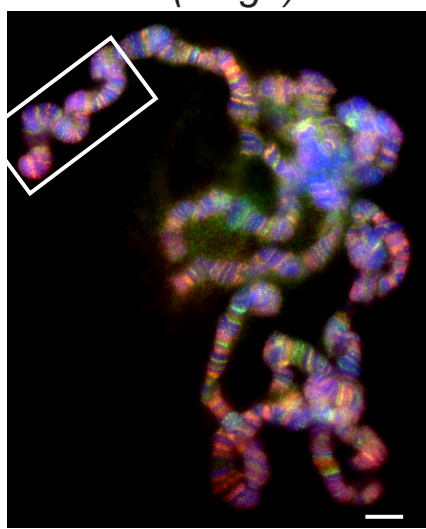

CP190, yH2Av, DAPI
CP190
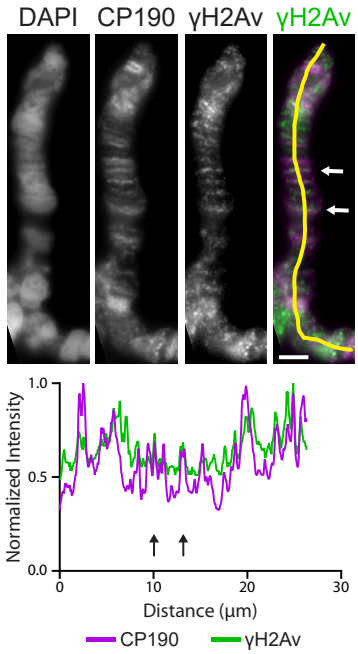

CP190 DAPI CP190 yH2Av $\mathrm{YH} 2 \mathrm{Av}$
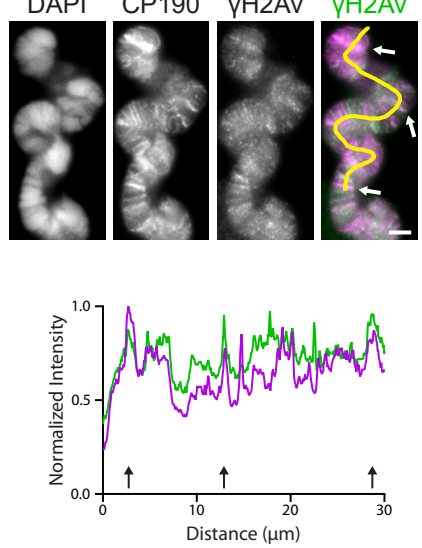

- CP190 - $\mathrm{\gamma H} 2 \mathrm{Av}$

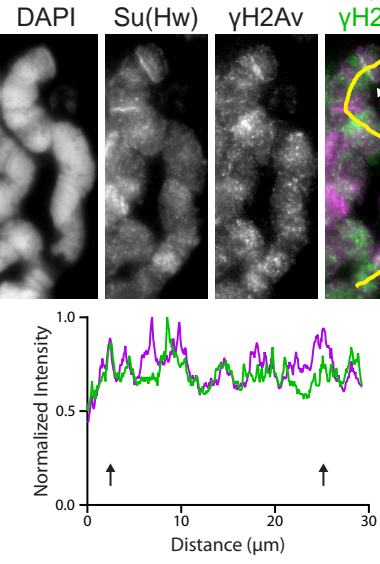

$-\mathrm{Su}(\mathrm{Hw})-\mathrm{yH} 2 \mathrm{Av}$

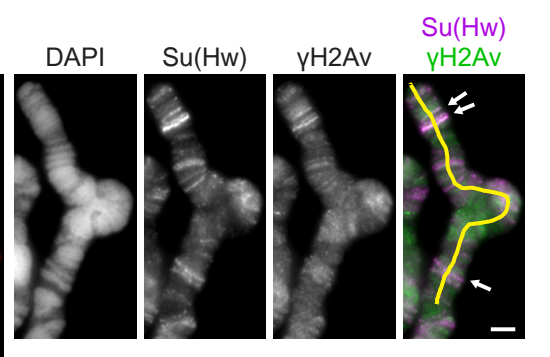

F

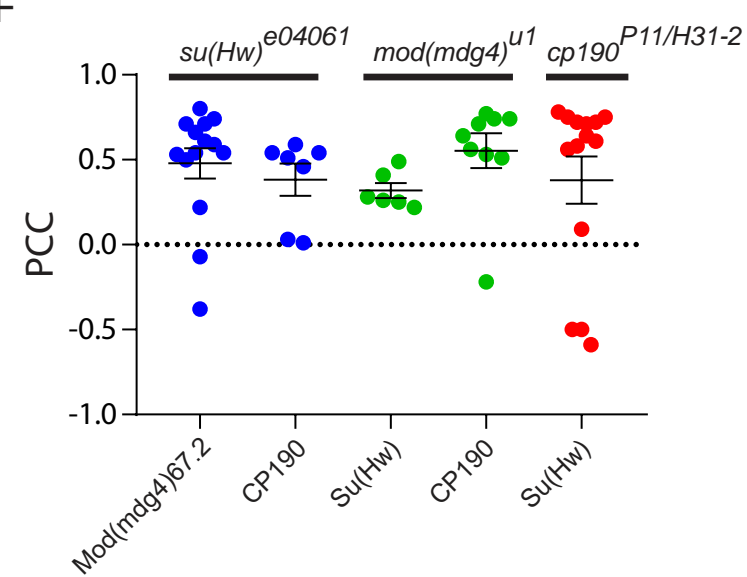

Figure 6. Phosphatase inhibition restores localization of $\gamma \mathrm{H} 2 \mathrm{Av}$ at insulator sites in insulator protein mutants. Shown are co-immunostains of polytene chromosomes from salivary glands treated with okadaic acid. A. Colocalization of $\gamma \mathrm{H} 2 \mathrm{Av}$ with Mod(mdg4)67.2 in su(Hw)e04061. B. Colocalization of $\mathrm{\gamma H} 2 \mathrm{Av}$ with $\mathrm{CP} 190$ in $s u(H w)^{e 04061}$. C. Colocalization of $\gamma \mathrm{H} 2 \mathrm{Av}$ with $\mathrm{Su}(\mathrm{Hw})$ in $\bmod (\operatorname{mdg} 4)^{u 1}$. D. Colocalization of $\gamma \mathrm{H} 2 \mathrm{Av}$ with $\mathrm{CP} 190 \mathrm{in} \bmod (\operatorname{mdg} 4)^{u 1}$. E. Colocalization of $\mathrm{\gamma H} 2 \mathrm{Av}$ with $\mathrm{Su}(\mathrm{Hw})$ in $c p 190^{\mathrm{P} 11 / \mathrm{H} 31-2}$. F. Pearson's Correlation Coefficient (PCC) for $\mathrm{\gamma H} 2 \mathrm{Av}$ signal with each insulator protein signal is plotted, with each point representing the polytene genome of each cell. Error bars represent one standard error of the mean. PCC values are grouped by genotype (red $=$ $s u(H w)^{e 04061}$, green $=\bmod (m d g 4)^{u 1}$, blue $\left.=c p 190^{p 11 / H 31-2}\right)$. Immunostaining results from polytene chromosome squashes are shown on the left in each panel. Magnified insets are shown to the right of each figure, corresponding to the white boxes in the figures on the left. Scale bars are $5 \mu \mathrm{m}$ in the figures and $2 \mu \mathrm{m}$ in the insets. Insets are shown as RGB merge, with DAPI on the blue channel, $\mathrm{\gamma H} 2 \mathrm{Av}$ on the green channel, and various insulator proteins on the red channel. Red and green channels are shown independently in grey scale and merged as magenta and green. Beneath the insets are linescans corresponding to the yellow lines in the merged insets. Linescan intensities were normalized by dividing each value by the maximum intensity recorded on each channel. 
resolution of insulator bodies during isotonic recovery, suggesting this is an essential part of the mechanism governing the cellular response to osmotic stress. It remains unclear whether $\mathrm{H} 2 \mathrm{Av}$ is phosphorylated after recruitment into insulator bodies or if chromatin-bound $\mathrm{H} 2 \mathrm{Av}$ is phosphorylated in response to osmotic stress before localizing to insulator bodies. A third possibility is that the $\mathrm{\gamma H} 2 \mathrm{Av}$ observed in insulator bodies originates from $\mathrm{H} 2 \mathrm{Av}$ that is already phosphorylated through normal metabolic activity. Further experiments will be necessary to discern which of these models is correct.

\section{H2Av Is Phosphorylated in gypsy Insulator Sites}

While a clear correlation is seen between H2Av phosphorylation and insulator binding proteins genome-wide, it is unclear if these interactions are a feature of insulators or sites where insulator proteins bind independently as transcription factors. For example, outside of the canonical gypsy insulator, $\mathrm{Su}(\mathrm{Hw})$ binds many sites in the genome alone or in conjunction with either CP190 or Mod(mdg4) (Kuhn-Parnell et al., 2008, Bushey et al., 2009, Soshnev et al., 2012, Soshnev et al., 2013). We therefore asked whether or not $\mathrm{H} 2 \mathrm{Av}$ phosphorylation is required specifically at gypsy insulator sites by looking at two classical examples, $y^{2}$ and $c t^{6}$ (Figure 9A). The former is an allele of the yellow $(y)$ gene in which a gypsy insulator site inserted into the region between enhancers for expression in the wing and body of the fly and the promoter, cutting off contact between the enhancers and promoter upstream of the yellow gene promoter and resulting in lowered expression of the yellow gene and ultimately lighter pigmentation of the adult fly (Harrison et al., 1989, Geyer and Corces, 1992). The latter example is an allele of the cut (ct) gene in which a gypsy insulator between the wing margin enhancer and cut promoter prevents expression of the cut gene in the developing wing. This decreases cut expression in cells of the wing margin, leading to a jagged appearance of the wing margin (Jack et al., 1991, Kim et al., 1996). As it has previously been demonstrated that a functional gypsy insulator complex composed of $\mathrm{Su}(\mathrm{Hw}), \operatorname{Mod}(\operatorname{mdg} 4) 67.2$, and CP190 is required for proper function of the insulator (Georgiev and Kozycina, 1996, Cai and Levine, 1997, Gause et al., 2001, Ghosh et al., 2001, Mongelard et al., 2002), these sites serve as known examples of genomic loci associated with binding of each of these components.

To determine if H2Av is phosphorylated at known gypsy sites, immunostaining of the $X$ polytene chromosomes of larvae carrying the $y^{2}$ and $c t^{6}$ alleles were performed in wild-type and mutant backgrounds. Notably, a strong colocalization was observed for phosphorylated $\mathrm{H} 2 \mathrm{Av}$ and each gypsy insulator component (Su(Hw), Mod(mdg4)67.2, and CP190) at both $y^{2}$ and $c t^{6}$ sites (Figure 8A). Strikingly, this colocalization is lost in $\bmod (\operatorname{mdg} 4)^{u 1}$ mutants (Figure $8 \mathrm{~B})$. $\mathrm{Su}(\mathrm{Hw})$ is still recruited to gypsy insulator sites in $\bmod (m d g 4)^{u 1}$ as expected based on previous reports of mod(mdg4) mutants (Ghosh et al., 2001, Melnikova, Kostyuchenko, et al., 2017); more significant, however, is the observation that $\mathrm{\gamma H} 2 \mathrm{Av}$ is no longer observed colocalizing with $\mathrm{Su}(\mathrm{Hw})$ at $y^{2}$ or $c t^{6}$. Likewise, $\mathrm{H} 2 \mathrm{Av}$ phosphorylation is no longer observed at either $y^{2}$ or $c t^{6}$ in the $s u(H w)^{e 04061}$ mutant background (Figure $8 \mathrm{C}$ ). The lack of Mod(mdg4)67.2 at y2 and ct6 in the absence of $\mathrm{Su}(\mathrm{Hw})$ agrees with previous reports, which implicate $\mathrm{Su}(\mathrm{Hw})$ as necessary for recruitment of $\mathrm{Mod}(\operatorname{mdg} 4) 67.2$ to gypsy loci (Ghosh et al., 2001, Melnikova, Kostyuchenko, Molodina, et al., 2018), while the lack of $\gamma \mathrm{H} 2 \mathrm{Av}$ implies that either $\mathrm{Su}(\mathrm{Hw})$ is directly needed to maintain $\mathrm{H} 2 \mathrm{Av}$ in a phosphorylated state or that a complete gypsy complex containing Mod(mdg4)67.2 is required. To expand on this question, polytene immunostaining from the

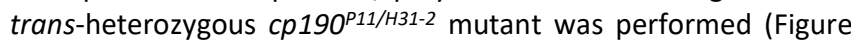
$8 D)$. This showed a decrease in the amount of $\mathrm{Su}(\mathrm{Hw})$ present at $y^{2}$ and $c t^{6}$, consistent with a previous report that found reductions in both $\mathrm{Su}(\mathrm{Hw})$ and $\mathrm{Mod}(\operatorname{mdg} 4) 67.2$ in the polytene chromatin of cp190 mutants, (Melnikova, Kostyuchenko, Molodina, et al., 2018). Similar to the mutations in $s u(H w)$ and $\bmod (\operatorname{mdg} 4)$, mutation of cp190 also significantly reduced levels of H2Av phosphorylation at the two gypsy loci examined. All together, these results indicate that reduction of any of the canonical gypsy insulator components is sufficient to disrupt $\mathrm{H} 2 \mathrm{Av}$ phosphorylation at these sites. This would argue against this relationship being limited to $\mathrm{Su}(\mathrm{Hw})$-only sites and supports the notion that H2Av phosphorylation is either promoted or stabilized by interactions with intact gypsy insulator complexes.

\section{H2Av Contributes to gypsy Insulator Function}

Our earlier results strongly suggest a correlation between $\mathrm{pH} 2 \mathrm{Av}$ and gypsy insulator components, including colocalization on polytene chromatin (Figures 1 and 8) and in insulator bodies (Figure 4). Some evidence for a functional relationship was found in the dissolution of insulator bodies (Figure 7) but it remained unclear if $\mathrm{H} 2 \mathrm{Av}$ plays any role in canonical insulator functions such as enhancer-blocking. Based on the correlations described above between gypsy insulator proteins and $\mathrm{\gamma H} 2 \mathrm{Av}$, we wondered whether or not mutation of His $2 A v$, the sole $\mathrm{H} 2 \mathrm{~A}$ variant gene found in the Drosophila genome (van Daal et al., 1988), would affect gypsy insulator function. As the null mutation of His $2 A v$ is homozygous lethal at the third instar larval stage (van Daal and Elgin, 1992), we were precluded from observing how a complete lack of H2Av would affect the yellow and cut phenotypes seen in adults. We therefore set up a series of crosses to examine how adult animals that carried heterozygous mutations for both His $2 \mathrm{Av}$ and $s u(H w)$.

The effects of these mutants on the $c t^{6}$ allele were measured by examining the wing margins. Wing margins in Drosophila contain mechanosensory bristles (Hartenstein and Posakony, 1989) that require expression of the cut gene for proper formation (Jack, 1985). Lack of cut expression results in decreased specification and differentiation of mechanosensory bristles and increased rates of cell death (Jack et al., 1991, Liu et al., 1991). The $c t^{6}$ phenotype is characterized by wings having an incomplete margin due to such defects, resulting in the nominal "cuts" in the wing which can be rescued to near wild type by mutations in gypsy insulator genes (Georgiev and Kozycina, 1996, Kim et al., 1996). Wing width was measured as a proxy for cut gene activity, as wings with the $c t^{6}$ phenotype are less wide due to the decreased cell proliferation associated with this phenotype. Width of the wing is calculated as the area divided by the feret diameter (a measure of length). Notably, flies that contained heterozygous mutations for both 
bioRxiv preprint doi: https://doi.org/10.1101/2021.02.23.432395; this version posted February 23, 2021. The copyright holder for this preprint (which was not certified by peer review) is the author/funder. All rights reserved. No reuse allowed without permission.

\begin{tabular}{|c|l|c|c|c|}
\hline Treatment & Media & $\begin{array}{c}\text { Number } \\
\text { of cells }\end{array}$ & $\begin{array}{c}\text { Cells with } \\
\text { bodies (\%) }\end{array}$ & Bodies/cell \\
\hline \multirow{3}{*}{ OA- } & Isotonic & 650 & 1.2 & 0.02 \\
& Hypertonic & 510 & 91.4 & 5.73 \\
& Recovery & 495 & 2.2 & 0.04 \\
\hline \multirow{3}{*}{ OA+ } & Isotonic & 596 & 0.5 & 0.01 \\
& Hypertonic & 487 & 88.9 & 4.63 \\
& Recovery & 596 & 63.1 & 3.11 \\
\hline
\end{tabular}

C

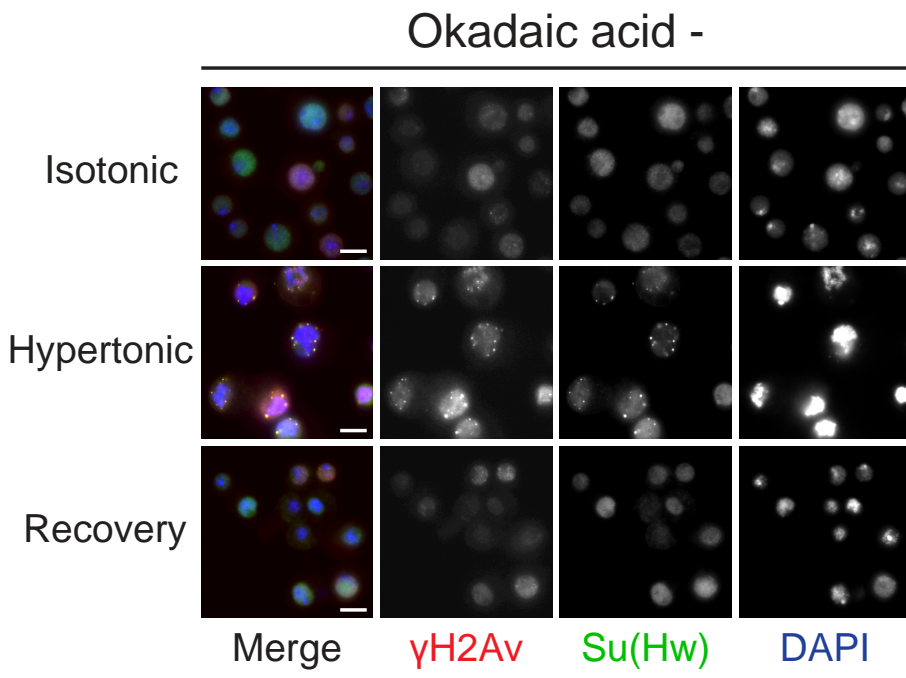

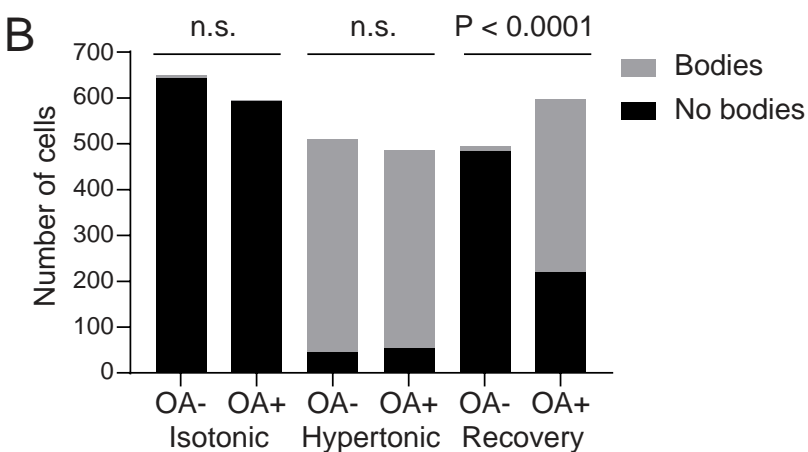

Okadaic acid +

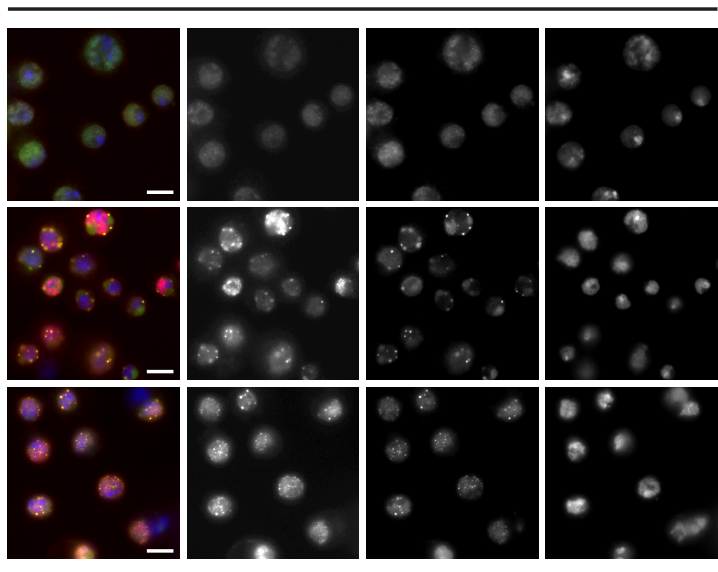

Merge

$\mathrm{yH} 2 \mathrm{Av}$

$\mathrm{Su}(\mathrm{Hw})$

DAPI

Figure 7. Phosphatase inhibition prevents recovery from insulator body formation after osmotic stress. A. Tabulated results from OA (okadaic acid) treatment in osmotic stress and recovery. B. Bar graph showing the number of cells displaying bodies (grey) and those not displaying bodies (black). OA- and OA+ treatments are shown for each osmotic condition. P-values were determined using Fisher's exact test. n. s. = not significant. C. Osmotic stress and recovery in the absence of okadaic acid (left) in the presence of okadaic acid (right). Representative images are shown of S2 cells in isotonic media, in hypertonic stress media, and recovering in isotonic media. Merged images are shown on the left, with each channel shown independently in greyscale. Scale bars represent $5 \mu$ m. Exposure times and contrast settings were kept constant between each sample. 
bioRxiv preprint doi: https://doi.org/10.1101/2021.02.23.432395; this version posted February 23, 2021. The copyright holder for this preprint (which was not certified by peer review) is the author/funder. All rights reserved. No reuse allowed without permission.

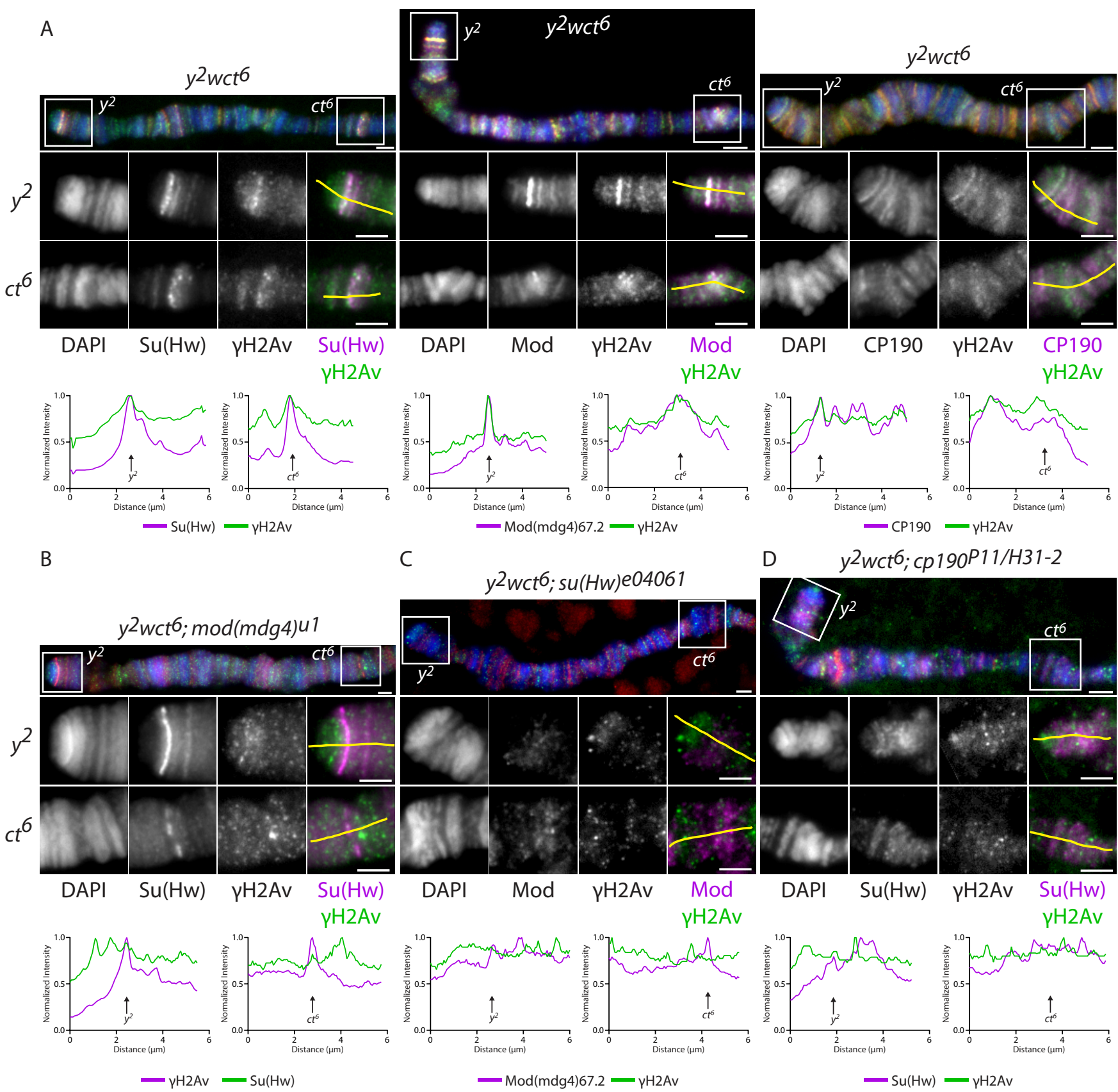

Figure 8. Phosphorylated H2Av is present at gypsy insulator sites. A. Colocalization between $\mathrm{HH} 2 \mathrm{Av}$ and $\mathrm{Su}(\mathrm{Hw})(\mathrm{left}), \mathrm{Mod}(\mathrm{mdg} 4) 67.2(\mathrm{center})$, and CP190 (right) in a wild type $y^{2} w c t^{6}$ background. B. Colocalization between $\mathrm{HH}_{2} \mathrm{Av}$ and $\mathrm{Su}(\mathrm{Hw})$ in a $\bmod (\operatorname{mdg} 4)^{u 1}$ homozygous background. C. Colocalization between $\gamma \mathrm{H} 2 \mathrm{Av}$ and

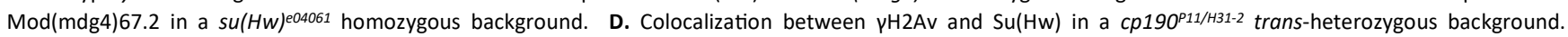
Immunostaining results are shown of the X polytene chromosome from wandering third instar larvae in the $y^{2} w c t^{6}$ background. Each panel shows an X chromosome, with the $y^{2}$ and $c t^{6}$ sites labelled and delineated with white boxes. Beneath these are insets showing the $y^{2}$ and $c t^{6}$ sites in detail. Insets are shown as RGB merge, with DAPI on the blue channel, $\gamma \mathrm{H} 2 \mathrm{Av}$ on the green channel, and various insulator proteins on the red channel. Red and green channels are shown independently in grey scale and merged as magenta and green. Beneath the insets are linescans corresponding to the yellow lines in the merged insets. Arrows represent the approximate sites of each gypsy locus. Scale bars are $2 \mu \mathrm{m}$ in the main figures and insets. Linescan intensities were normalized by dividing each value by the maximum intensity recorded on each channel. 
His $2 A v^{810}$ and $s u(H w)^{e 04061}$ displayed wider wings, while both single heterozygotes showed varying degrees of cuts in the margin (Figure 9B). To verify this finding, the null $s u(H w)^{v}$ allele was next tested in conjunction with $H i s 2 A v^{810}$. Consistent with the $H i s 2 A v^{810} / \mathrm{su}(\mathrm{Hw})^{e 04061}$ genotype, $\mathrm{His} 2 \mathrm{Av} v^{810} / \mathrm{su}(\mathrm{Hw})^{v}$ double heterozygotes had significantly wider wings than either single heterozygote (Figure 9B). This increased suppression of gypsy insulator phenotypes in $s u(H w)$ heterozygous backgrounds implies that $\mathrm{H} 2 \mathrm{Av}$ is involved in gypsy insulator function.

In order to determine if this requirement for H2Av in gypsy insulator function is specific to the cut gene or if it is a general mechanism for gypsy insulators, we tested the effect of His2Av mutation on the $y^{2}$ phenotype. Effects of these mutations on the $y^{2}$ allele were tested by measuring the degree of pigmentation in the wings and in the darkened A5 tergite found in male flies. Heterozygous HisAv $v^{810}$ or su(Hw) ${ }^{e 04061}$ mutations by themselves have little effect on expression of yellow in the abdomen (Figure 9C). In contrast to this, abdomens in the double heterozygous $H i s 2 A v^{810} / s u(H w)^{e 04061}$ mutants were significantly darker than the single heterozygous mutants (Figure 9C). This implies a reduction in the enhancer-blocking capacity of the gypsy insertion upstream of yellow and suggests a functional role for $\mathrm{H} 2 \mathrm{Av}$ in gypsy insulator function. In order to exclude the possibility that second-site mutations in the $s u(H w)^{e 04061}$ background were influencing this rescue, we crossed the null $s u(H w)^{v}$ allele with the His2Av ${ }^{810}$ mutant. Flies doubly heterozygous for $s u(H w)^{v}$ and $H i s 2 A v^{810}$ showed a statistically significant increase in pigmentation compared to the His $2 A v^{810}$ heterozygote, but not the single $s u(H w)^{v}$ mutant (Figure $\left.9 \mathrm{C}\right)$. This discrepancy between $s u(H w)^{e 04061}$ and $s u(H w)^{v}$ may be due to mutation of the neighboring Rpll15 gene (an RNA Pol Il subunit) in the $s u(H w)^{v}$ chromosome (Harrison et al., 1992), which may have an epistatic effect on this phenotype. Taken together, these findings represent the first evidence for the requirement of a histone protein in Drosophila insulator function and may hint at a mechanism for how insulators work. There is a correlation between the lack of a balancer chromosome and the rescues seen in this assay. The lack of a balancer is unlikely to be a causative factor in the rescues, as the TM6B and TM6C balancers present in the single heterozygotes carry the ebony ${ }^{1}$ marker, which significantly increases the degree of pigmentation (Wittkopp et al., 2002), and the single heterozygotes are lighter than the transheterozygotes.

\section{YH2Av colocalizes with $\mathrm{Su}(\mathrm{Hw})$ at TAD boundaries}

Next, given the previous observations indicating that $\mathrm{\gamma H} 2 \mathrm{Av}$ and $\mathrm{Su}(\mathrm{Hw})$ sites colocalize in polytene chromosomes, we asked whether the colocalization of both proteins is also supported at the molecular level by Chromatin Immunoprecipitation (ChIP) experiments. To answer this question, we used publicly available ChIP data for $\mathrm{YH} 2 \mathrm{Av}$ and Su(Hw) from Kc167 cells (SRX1299942 SRA) (Li et al., 2016). We used SeqMiner to compare the two ChIPseq datasets by heatmap analysis. The peak summits for $\mathrm{\gamma H} 2 \mathrm{Av}$ were taken as the reference coordinate for a heatmap and profile analysis comparing $\mathrm{Su}(\mathrm{Hw})$ and $\mathrm{yH} 2 \mathrm{Av}$ distributions (Figure 10A). The profile of mean read densities for both proteins also shows a significant overlap between $\mathrm{Su}(\mathrm{Hw})$ and $\mathrm{\gamma H} 2 \mathrm{Av}$ peaks at $\mathrm{\gamma H} 2 \mathrm{Av}$ peaks flanked by $10,000 \mathrm{bp}$ (Figure 10B). These results support our observation that the genomic distribution of $\mathrm{Su}(\mathrm{Hw})$ and $\mathrm{yH} 2 \mathrm{Av}$ overlap in polytene chromosomes.

Because insulator proteins are frequently associated with the boundaries of Topologically Associating Domains (TADs), we next asked whether $\gamma \mathrm{H} 2 \mathrm{Av}$ is also enriched at TAD boundaries. To address this question, we used publicly available data and obtained a map of all Drosophila TADs as determined by $\mathrm{Hi}-\mathrm{C}$ using Drosophila Kc167 cells (Ramirez et al., 2018). We used this data to generate a BED file consisting of $1,000 \mathrm{bp}$ DNA fragments containing all TAD boundaries in the Drosophila genome flanked by $500 \mathrm{bp}$ at both sites of the boundary. The $1 \mathrm{~kb}$ TAD boundary BED file was used as a reference coordinate for a heatmap comparing the density distribution of nucleosomes containing the histone variant $\mathrm{H} 2 \mathrm{Av}$, either homotypic ( $\mathrm{H} 2 \mathrm{Av}$ Hom) or heterotypic ( $\mathrm{H} 2 \mathrm{Av}$ $\mathrm{Het}$ ), as well as the densities of $\mathrm{yH} 2 \mathrm{Av}$ and $\mathrm{Su}(\mathrm{Hw})$ in the Drosophila genome (Figure $10 \mathrm{C}$ ). The distribution of $\mathrm{H} 2 \mathrm{Av}$ nucleosomes was obtained from a previous study (Weber et al., 2010). The heatmap analysis shows there is a strong association between the density distribution of $\mathrm{H} 2 \mathrm{Av}$ nucleosomes and TAD boundaries (Figure 10C). Generally, we found homotypic H2Av nucleosomes are enriched at the boundaries, whereas heterotypic nucleosomes have a significant density drop at TAD boundaries.

Interestingly, enrichment in $\mathrm{\gamma H} 2 \mathrm{Av}$ and $\mathrm{Su}(\mathrm{Hw})$ is also observed at the TAD boundaries (Figure 10C). Signal enrichment can be grouped in two major clusters, where the most significant difference is the relative enrichment of $\mathrm{Su}(\mathrm{Hw})$ and $\mathrm{\gamma H} 2 \mathrm{Av}$ at the boundaries (Figure 10D, E). In cluster 2, heterotypic H2Av and $\gamma \mathrm{H} 2 \mathrm{Av}$ have similar enrichment levels through the DNA flanking the boundary. At the center of the boundary, however, is the homotypic instead of the heterotypic $\mathrm{H} 2 \mathrm{Av}$ that has an enrichment similar to that of $\mathrm{\gamma H} 2 \mathrm{Av}$, with both intensity profiles (H2Av Hom and $\mathrm{\gamma H} 2 \mathrm{Av}$ ) significantly more elevated than in the flanking DNA (Figure 10E). Interestingly, Cluster 1 has the opposite pattern. In cluster 1 , homotypic $\mathrm{H} 2 \mathrm{Av}$ and $\mathrm{\gamma H} 2 \mathrm{Av}$ have similar enrichment densities through the DNA flanking the boundary and remain elevated at the boundary center. However, $\gamma \mathrm{H} 2 \mathrm{Av}$ and $\mathrm{Su}(\mathrm{Hw})$ intensities are similar and higher than that of the homotypic and heterotypic H2Av nucleosomes (Figure 10D).

The functional significance of the association of $\gamma \mathrm{H} 2 \mathrm{Av}$ with TAD boundaries is intriguing. Interestingly, we found that the association of $\mathrm{YH} 2 \mathrm{Av}$ with TAD boundaries is very similar to that of $\mathrm{Su}(\mathrm{Hw})$ and other insulator proteins in that is found as an enriched peak. One such example of this is a TAD boundary that flanks a TAD containing the homeotic gene Abdominal $A(A b d-A)$. Like other boundaries associated with the homeobox gene cluster (Postika et al., 2018, Ozdemir and Gambetta, 2019), this boundary is enriched in the insulator proteins $\mathrm{Su}(\mathrm{Hw})$, Modifier of Mdg4, CP190, CTCF, and HIPP1. Here, we show this boundary is equally enriched in $\mathrm{\gamma H} 2 \mathrm{Av}$ (Figure 10F). Moreover, when observing the distribution of $\mathrm{H} 2 \mathrm{Av}$ nucleosomes at this site, it appears that the $\mathrm{\gamma H} 2 \mathrm{Av}$ peak does not colocalize with a nucleosome, suggesting the possibility that $\mathrm{H} 2 \mathrm{Av}$ may be non-nucleosomal. In another example, two boundaries flank a TAD that contains the developmentally regulated pair rule gene eve (Figure 10G). Each boundary is enriched in $\mathrm{\gamma H} 2 \mathrm{Av}$; however, in the left boundary the $\mathrm{\gamma H}_{2} \mathrm{Av}$ peak 
A
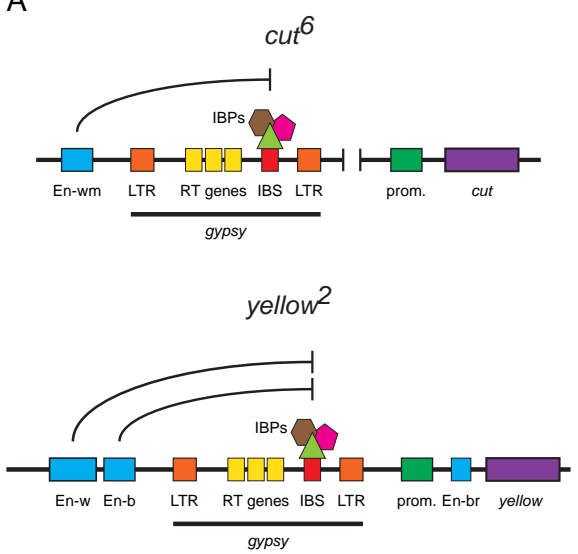

B

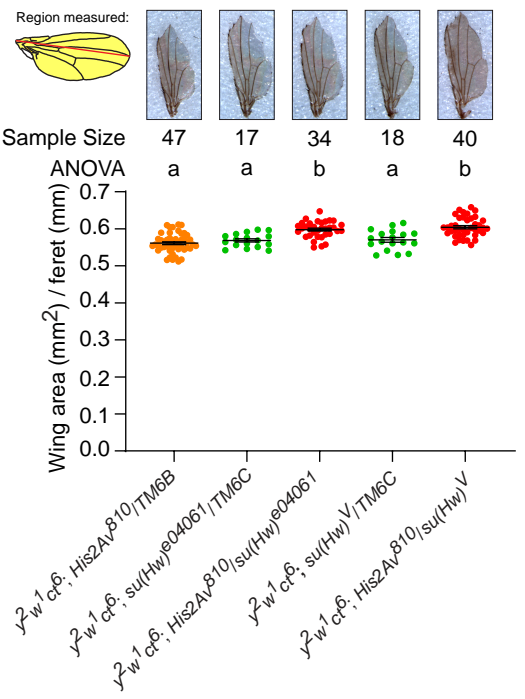

C

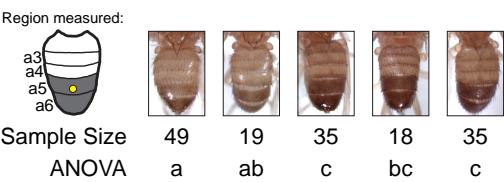

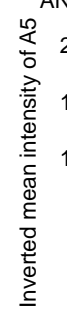
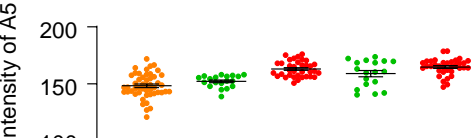

100

50

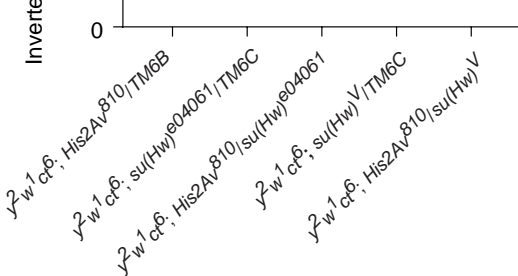

Figure 9. H2Av contributes to gypsy insulator function. A. Illustration of the upstream regulatory elements found in the $c t^{6}$ and $y^{2}$ alleles. Gene coding sequences (purple) are regulated by coordinated contacts between enhancers (blue) and promoters (green). In each case, the gypsy retrotransposon (denoted with the thick black bar) has been inserted between a promoter and at least one enhancer. LTR = long terminal repeats, RT genes = retrotransposon genes (gag, pol, and env), IBS $=$ insulator binding site, IBPs = insulator binding proteins $(\mathrm{Su}(\mathrm{Hw}), \operatorname{Mod}(\mathrm{mdg} 4) 67.2$, and $\mathrm{CP} 190)$. When the IBS is bound by a complete insulator complex, it interrupts communication between the promoter and distal enhancers. In cut $t^{6}$ this prevents expression of cut by the wing margin enhancer (En-wm), while in yellow ${ }^{2}$ the insulator prevents expression of yellow directed by the wing enhancer (En-w) and the body enhancer (En-b) but not the bristle enhancer (En-br) between the yellow promoter and transcription start site. Genomic distances are not drawn to scale. B. The average length of wings along the feret axis was used as a metric for the cut phenotype. Wing area in $\mathrm{mm}^{2}$ (yellow shaded area) was divided by feret diameter (red line). Examples of wings are displayed above their respective genotypes. C. $y^{2}$ phenotype scoring in the male abdomen. The illustration on the left shows the male abdomen with abdominal segments 3-6 labelled. A circular ROI (yellow circle) was sampled from the a5 tergite of each male to determine the degree of pigmentation. The graph depicts the mean pixel intensity from individual flies by genotype. Example pictures of abdomens are displayed above their respective genotypes. In B and C, green dots represent su( $H w)$ heterozygotes arising from each cross, orange dots represent $H i s 2 A v^{810}$ heterozygotes, and red dots indicate flies doubly heterozygous for His $2 A v^{810}$ and $s u(H w)$. Above each data set is the sample size and single letter statistical codes from an ANOVA performed with Dunnett's T3 multiple comparisons, with significant diffe rences at $P \leq 0.05$. 
overlaps at least partially with the nucleosomal $\mathrm{H} 2 \mathrm{Av}$, whereas in the right boundary the $\mathrm{\gamma H} 2 \mathrm{Av}$ peak seems to be non-nucleosomal as well. This particular boundary corresponds to the well characterized Homie insulator (Fujioka et al., 2013). These findings show that the distribution of $\mathrm{\gamma H} 2 \mathrm{Av}$ in the Drosophila genome display all the properties of insulator proteins, suggesting that $\mathrm{\gamma H} 2 \mathrm{Av}$ may be required for insulator function.

\section{Discussion}

Our results have demonstrated a clear relationship between the phosphorylated histone variant $\mathrm{\gamma H} 2 \mathrm{Av}$ and chromatin insulator proteins. $\mathrm{YH} 2 \mathrm{Av}$ colocalizes with $\mathrm{Su}(\mathrm{Hw})$ insulator complexes throughout the genome, and both are enriched at TAD boundaries. Disruption of insulator complex formation prevents stable phosphorylation of $\mathrm{H} 2 \mathrm{Av}$ that can be overcome by phosphatase inhibition. Importantly, we provide evidence that this histone variant is involved in insulator activity, as dephosphorylation of $\mathrm{\gamma H} 2 \mathrm{Av}$ is necessary for dissolution of insulator bodies and reducing the genetic dose of $\mathrm{H} 2 \mathrm{Av}$ in a sensitized $s u(\mathrm{HW})$ heterozygous mutant background partially rescues gypsy insulator phenotypes.

Chromatin insulator proteins were initially characterized by their enhancer-blocking properties and their ability to prevent the spread of heterochromatin, and more recently by their role in large-scale genome organization (Harrison et al., 1989, Geyer and Corces, 1992, Bell et al., 1999, Bushey et al., 2009). In addition to these canonical properties, however, our lab has uncovered roles of insulators in other aspects of cell metabolism including the osmotic stress response (Schoborg et al., 2013) and genome stability (Hsu et al., 2019). It is now established that mutation of the only insulator protein found in humans, CTCF, predisposes cells to cancer formation (Docquier et al., 2005, Kemp et al., 2014, Guo et al., 2018) through increased rates of unrepaired DNA damage. Despite this knowledge, the mechanisms linking insulator activity to the maintenance of genome stability remain enigmatic. Here, we report a functional relationship between a Drosophila insulator and $\mathrm{H} 2 \mathrm{Av}$, the sole histone $\mathrm{H} 2 \mathrm{~A}$ variant in fruit flies. $\mathrm{H} 2 \mathrm{Av}$ in Drosophila performs functions associated with both the mammalian histone variants $\mathrm{H} 2 \mathrm{AX}$ and $\mathrm{H} 2 \mathrm{AZ}$ (Baldi and Becker, 2013), and misregulation of H2Av in Drosophila is associated with severe developmental phenotypes including nuclear falling in embryos (Li et al., 2014), the formation of necrotic tumors in the larval lymph gland (Grigorian et al., 2017) and an inability to maintain stem cell populations in adult tissues (Morillo Prado et al., 2013). Like $H 2 A X, H 2 A v$ is phosphorylated in response to DNA double strand breaks (DSBs) and serves as a chromosomal mark to recruit DNA repair proteins (Madigan et al., 2002, Joyce et al., 2011).

Our initial experiments in Drosophila polytene chromosomes revealed a striking correlation between the binding sites of phosphorylated $\mathrm{H} 2 \mathrm{Av}$ and insulator proteins at gypsy insulator sites (Figure 8) and at $\mathrm{Su}(\mathrm{Hw})$ sites elsewhere in the genome (Figures 1, 10). Importantly, this colocalization depends on having a complete and stable $\mathrm{Su}(\mathrm{Hw})$ insulator complex, as mutating any of the three core insulator components reduces the coincidence of $\gamma \mathrm{H} 2 \mathrm{Av}$ signals with the remaining insulator proteins (Figure 3). Some insight into the mechanism behind this phenomenon comes from experimental inhibition of PP2A, the phosphatase responsible for dephosphorylating $\mathrm{\gamma H} 2 \mathrm{Av}$ after resolution of double strand breaks (Merigliano et al., 2017). We found that the amount of $\gamma \mathrm{H} 2 \mathrm{Av}$ in undamaged polytene chromosomes increases after PP2A inhibition. This was as expected due to the known role of PP2A in regulating $\mathrm{H} 2 \mathrm{Av}$ phosphorylation. Surprisingly, the amount of $\mathrm{Su}(\mathrm{Hw})$ bound to the polytene chromosomes also significantly rose when tissues were treated with the PP2A inhibitor (Figure 5). This seems to imply that gypsy insulator complex formation or stability is driven at least in part by the phosphorylation state of $\mathrm{H} 2 \mathrm{Av}$, although we cannot rule out the potential impact that other PP2A substrates may have on insulator complexes. Indeed, we show that inhibition of PP2A rescues gypsy insulator complex formation in tissues missing one of the three core insulator binding proteins (Figure 6). This supports the notion that $\mathrm{Su}(\mathrm{Hw})$ insulators are stabilized by the presence of phosphorylated H2Av.

To further explore the relationship between insulator binding proteins and $\mathrm{\gamma H} 2 \mathrm{Av}$, we performed immunostains on polytene chromosomes in the background of mutations in genes encoding for insulator proteins. We observed significant reductions of $\mathrm{\gamma H} 2 \mathrm{Av}$ signal in $s u(H w)^{e 04061}$ and $c p 190^{P 11 / H 31-2}$ (Figure 2), suggesting a relationship between $\mathrm{\gamma H} 2 \mathrm{Av}$ and gypsy insulator components in terms of recruitment to the chromatin. Notably, the reduction of $\mathrm{\gamma H} 2 \mathrm{Av}$ in $\mathrm{su}(\mathrm{Hw})^{e 04061}$ was more extreme than in other mutants. From this we postulate that the interaction between $\mathrm{yH} 2 \mathrm{Av}$ and $\mathrm{Su}(\mathrm{Hw})$ insulator sites may be largely mediated through interactions with $\mathrm{Su}(\mathrm{Hw})$ itself, although interactions with Mod(mdg4)67.2 and CP190 may also contribute to the overall stability of the complex. A large fraction of $\mathrm{Su}(\mathrm{Hw})$ binding sites do not include Mod(mdg4)67.2 or CP190 based on chromatin immunoprecipitation data (Negre et al., 2010). It will thus be informative for future experiments to determine whether or not the presence of $\mathrm{H} 2 \mathrm{Av}$ or $\mathrm{\gamma H} 2 \mathrm{Av}$ is required for insulator activity or transcriptional regulation at other loci bound by $\mathrm{Su}(\mathrm{Hw})$.

Our results to this point showed numerous correlations between gypsy insulators and $\mathrm{\gamma H} 2 \mathrm{Av}$ in chromatin, but it remained unclear if there was a functional relationship. Therefore, our final round of experiments sought to determine if $\mathrm{\gamma H} 2 \mathrm{Av}$ influences insulator function. These experiments are limited by the lethality of adults $\mathrm{H} 2 \mathrm{Av}$ homozygous mutant, but one key finding is the partial rescue of the $c t^{6}$ phenotype in $s u(H w)^{e 04061} / H i s 2 A v^{810}$ double heterozygotes (Figure 9). Significant increases in pigmentation in the abdomens of male flies were also found in this genotype in the $y^{2}$ background, and the $c t^{6}$ phenotype rescue was replicated in the $s u(H w)^{v} / H i s 2 A v^{810}$ background (Figure 9). This demonstrates that $\mathrm{H} 2 \mathrm{Av}$ influences the activity of insulator complexes in multiple tissues and therefore could be considered an insulator protein itself.

Our findings point to a model in which gypsy insulator components and $\mathrm{\gamma H}_{2} \mathrm{Av}$ stabilize each other in the chromatin (Figure $10 \mathrm{H}$ ). Based on our results and the fact that $\mathrm{Su}(\mathrm{Hw})$ makes direct interactions with DNA while Mod(mdg4)67.2 and CP190 are recruited by interactions with $\mathrm{Su}(\mathrm{Hw})$ (Harrison et al., 1993, Gdula et al., 1996, Ghosh et al., 2001, Pai et al., 2004, Melnikova, Kostyuchenko, et al., 2017), we propose that $\mathrm{Su}(\mathrm{Hw})$ is the main 
bioRxiv preprint doi: https://doi.org/10.1101/2021.02.23.432395; this version posted February 23, 2021. The copyright holder for this preprint (which was not certified by peer review) is the author/funder. All rights reserved. No reuse allowed without permission.

A

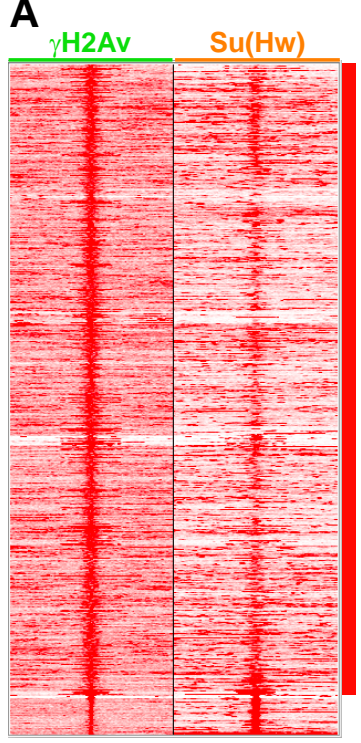

F

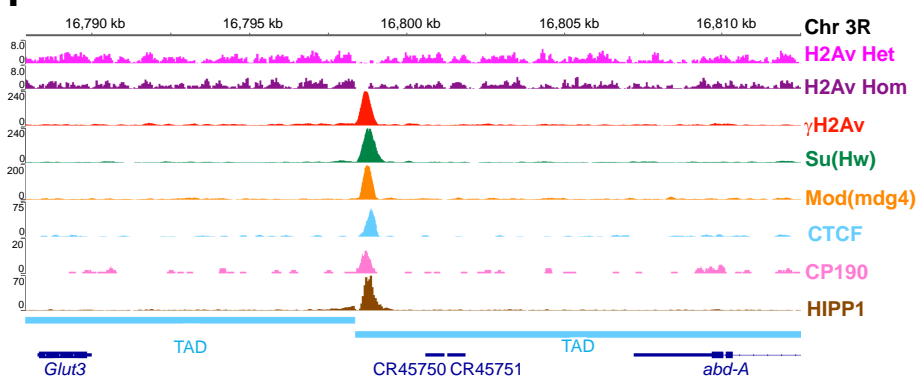

H

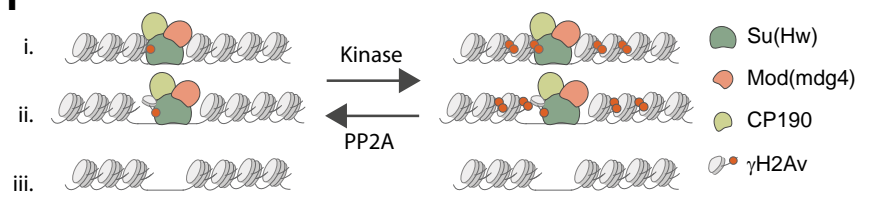

C

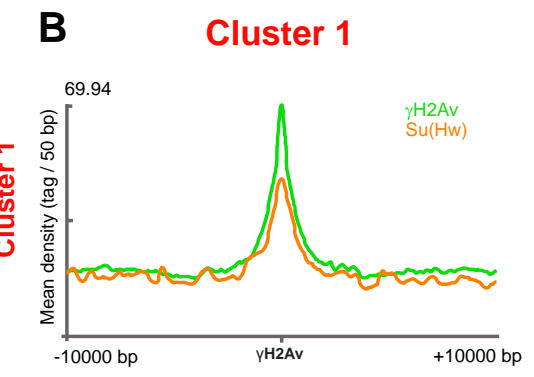

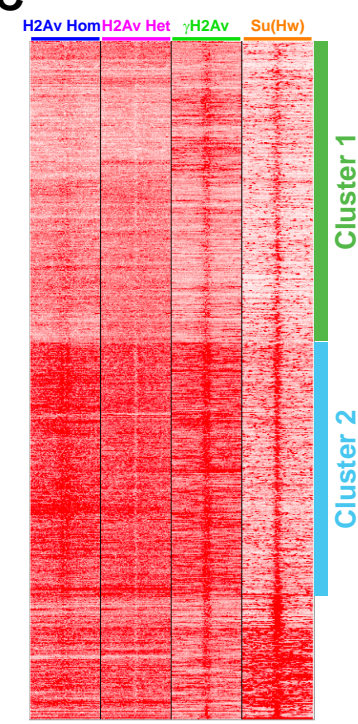

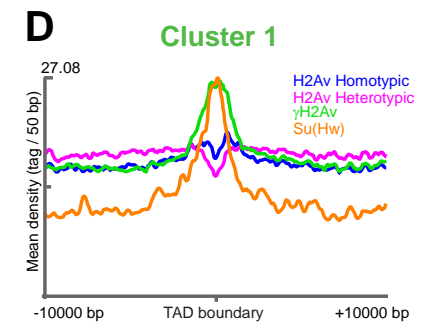

G
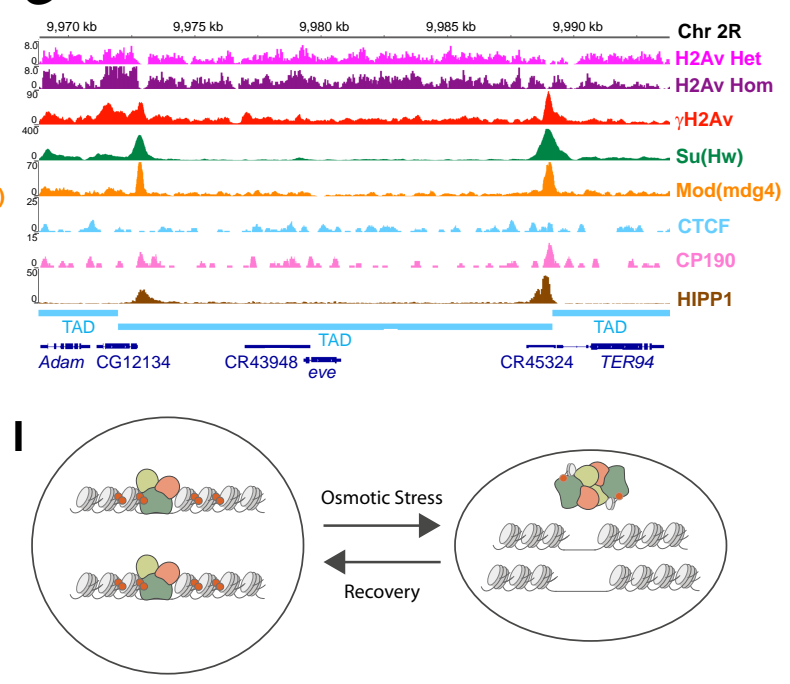

Figure 10: Phosphorylated H2Av associates with TAD boundaries. A. Heatmap comparing the intensity distributions of $\gamma \mathrm{H} 2 \mathrm{Av}$ and $\mathrm{Su}(\mathrm{Hw}) \mathrm{using} \mathrm{\gamma H} 2 \mathrm{Av}$ peaks as reference. B. Mean read density profiles of $\mathrm{\gamma H} 2 \mathrm{Av}$ and $\mathrm{Su}(\mathrm{Hw})$ centered at $\mathrm{\gamma H} 2 \mathrm{Av}$ peaks. C. Heatmap comparing the intensity distributions of $\mathrm{H} 2 \mathrm{Av}$ nucleosomes, $\gamma \mathrm{H} 2 \mathrm{Av}$, and $\mathrm{Su}(\mathrm{Hw})$ using TAD boundaries as a reference. D. and E. Mean read density profiles of two major clusters from heatmap in C, centered at TAD boundaries. F. Peak profile of insulator proteins, including $\mathrm{\gamma H} 2 \mathrm{Av}$ and the $\mathrm{H} 2 \mathrm{Av}$ nucleosome distribution at the left boundary of the $A b d-A \mathrm{TAD}$. G. Peak profile of insulator proteins, including $\mathrm{\gamma H} 2 \mathrm{Av}$ and the $\mathrm{H} 2 \mathrm{Av}$ nucleosome distribution at the Homie insulator flanking the pair rule gene eve. $\mathbf{H}$. This model shows the interaction between gypsy insulator components and $\mathrm{H} 2 \mathrm{Av}$ phosphorylation. We propose that binding of the $\mathrm{Su}(\mathrm{Hw})$ insulator complex promotes phosphorylation of neighboring $\mathrm{H} 2 \mathrm{Av}$. This stabilizes binding of the insulator complex and promotes spread of the $\mathrm{H} 2 \mathrm{Av}$ phosphorylation mark through neighboring nucleosomes. (i.) Case in which Su(Hw) complexes interact with nucleosomal $\gamma \mathrm{H} 2 \mathrm{Av}$. (ii.) Case in which $\mathrm{Su}(\mathrm{Hw}$ ) complexes interact with non-nucleosomal $\mathrm{\gamma H} 2 \mathrm{Av}$. (iii.) Case in which Su(Hw) is absent and phosphorylation of $\mathrm{H} 2 \mathrm{Av}$ is significantly reduced. I. During osmotic stress, insulator complexes leave chromatin and form insulator bodies. These bodies contain $\mathrm{\gamma H} 2 \mathrm{Av}$ that must be dephosphorylated during recovery so that insulator proteins can return to their genomic binding sites. 
site of the interaction with $\mathrm{yH} 2 \mathrm{Av}$ and that $\mathrm{Mod}(\operatorname{mdg} 4) 67.2$, and potentially CP190, stabilize this interaction. Further biochemical analysis will be required to determine if $\mathrm{\gamma H}_{2} \mathrm{Av}$ is in direct physical contact with $\mathrm{Su}(\mathrm{Hw})$ and, if so, which domains are the contact points between these proteins. Another possibility is an indirect association between $\mathrm{\gamma H} 2 \mathrm{Av}$ and $\mathrm{Su}(\mathrm{Hw})$, possibly via another yet unknown protein. Interactions with RNA may be another mechanism by which these proteins associate based on the recent finding that Shep RNA is required for gypsy insulator function (Chen et al., 2019). Direct binding with Mod(mdg4)67.2 or CP190 cannot be ruled out either, though such interactions are likely to have less effect on $\mathrm{yH}_{2} \mathrm{Av}$ recruitment than that with $\mathrm{Su}(\mathrm{Hw})$, based on results from the $c t^{6}$ and $y^{2}$ phenotypic rescue experiment (Figure 9).

Our model also proposes that $\mathrm{\gamma H} 2 \mathrm{Av}$ localizes in insulator bodies that form as a result of osmotic stress (Figure 10I). The mechanism remains unknown with possibilities including disassembly of the complex and reassociation as nucleoplasmic bodies or the entire complex translocating from chromatin into insulator bodies. It is not yet clear, however, if the $\mathrm{\gamma H} 2 \mathrm{Av}$ found in insulator bodies comes from the same population as found in chromatin or if these proteins are recruited from the non-chromatin bound nucleoplasmic $\mathrm{H} 2 \mathrm{Av}$ population.

An interaction between $\mathrm{Su}(\mathrm{Hw})$ and nucleosomes was recently reported in which gypsy insulators serve to position nucleosomes and establish regular nucleosome phasing (Baldi et al., 2018). Our observations may relate to this finding if such $\mathrm{Su}(\mathrm{Hw})$-based nucleosome positioning interactions depend on the presence of $\mathrm{yH}_{2} \mathrm{Av}$. Another possibility is a potential relationship between contacts with gyspy insulator proteins and whether neighboring nucleosomes are heterotypic or homotypic for this histone (Weber et al., 2010). Our ChIP-seq analysis shows enrichment for homotypic $\mathrm{H} 2 \mathrm{Av}$ along with $\mathrm{Su}(\mathrm{Hw})$ and other insulator proteins at TAD borders (Figure 10). This raises the question of whether gypsy insulator proteins affect the composition of nearby nucleosomes, or conversely, if these insulator proteins are preferentially recruited to homotypic nucleosomes. It is also possible that $\mathrm{H} 2 \mathrm{Av}$ is acting as an insulator protein outside of a nucleosome, as ChIPseq peaks enriched for $\mathrm{\gamma H} 2 \mathrm{Av}$ and insulator proteins do not necessarily align to nucleosomes (Figure 10). While the mechanistic relationship between these groups of proteins remains unknown, our data add to a growing consensus that interactions between histone proteins and insulator proteins are required for cellular homeostasis and maintenance of genome integrity.

Recent results from mammalian cell culture studies highlight an interaction between $\mathrm{H} 2 \mathrm{AZ}$, the histone variant associated with transcriptional regulation (Giaimo et al., 2019), and CTCF, the sole insulator protein in mammals (Wen et al., 2020). As Drosophila $\mathrm{H} 2 \mathrm{Av}$ serves orthologous functions to both mammalian $\mathrm{H} 2 \mathrm{AZ}$ and $\mathrm{H} 2 \mathrm{AX}$, our findings regarding insulators and the early DSB marker $\mathrm{YH} 2 \mathrm{Av}$ may be relevant to human health as CTCF is frequently mutated in various cancers (Kemp et al., 2014, Canela et al., 2017, Guo et al., 2018). Mutation of CTCF in Drosophila is associated with instability in ribosomal DNA found in the nucleolus (Guerrero and Maggert, 2011). If the relationship between insulator proteins and the DNA damage repair pathway is conserved from flies to humans, testing for interdependence of these components may provide clinically useful information. Indeed, it was recently described that CTCF sets the boundaries for phosphorylated H2AX spreading in human cell culture (Natale et al., 2017) and that CTCF and $\gamma \mathrm{H} 2 \mathrm{AX}$ are both recruited to sites of DSBs in mouse embryonic fibroblasts (MEFs) (Lang et al., 2017). Unlike our findings with Su(Hw), CTCF depletion in MEFs increases $\mathrm{H} 2 \mathrm{AX}$ phosphorylation but, similar to $\mathrm{Su}(\mathrm{Hw})$ depletion in flies, induces genome instability (Lang et al., 2017), possibly indicating a different mechanism linking CTCF to H2AX phosphorylation. The link between DNA damage repair and insulator proteins is further corroborated by the recent finding that TAD boundary strength and CTCF insulation strength both increase in an ATM-dependent manner in human cell culture after X-rayinduced DNA damage (Sanders et al., 2020).

Other questions generated from our results here will need to be addressed in future experiments. One example is determining how $\mathrm{H} 2 \mathrm{Av}$ or its phosphorylated form affect other phenotypes associated with lack of $s u(H w)$ expression. Importantly, su(Hw) has been shown to regulate genome stability (Lankenau et al., 2000) and we recently described the presence of chromosomal aberrations in actively dividing neuroblasts of $s u(\mathrm{Hw})$-deficient larvae (Hsu et al., 2020). How these aberrations arise is still unclear, however, our finding here that $\mathrm{su}(\mathrm{Hw})$ homozygotes show significantly less phosphorylated $\mathrm{H} 2 \mathrm{Av}$ may shed light on a mechanism. Cells that are unable to phosphorylate H2Av in response to DNA damage may be unable to recruit essential DNA damage repair proteins to the site of DSBs. If left alone, these unrepaired DSBs become obvious candidates for the source of the chromosomal aberrations seen in larval neuroblasts. Further studies will address this question, along with examining the role of $\mathrm{Su}(\mathrm{Hw})$ in repair of induced DNA damage, as well as the role of $\mathrm{\gamma H}_{2} \mathrm{Av}$ in boundary function and genome organization. Our results so far provide a foundation for understanding how the interplay between chromatin insulators and histones influences gene regulation and genome stability.

\section{Acknowledgements}

We would like to thank former members of the Labrador lab, including Dr. Emily Stow, for collaboration and discussion. We thank Dr. Rachel Patton McCord for critical review of the manuscript. Stocks obtained from the Bloomington Drosophila Stock Center (NIH P40OD018537) were used in this study. S2 cell culture was obtained from the Drosophila Genomics Resource Center (NIH 2P40OD010949).

\section{Competing Interests}

The authors declare no competing or financial interest. 


\section{Author contributions}

Conceptualization: J.R.S., R.A., M.L.; Data curation: J.R.S., R.A.; Formal analysis: J.R.S., B.A., M.L.; Funding acquisition: M.L.; Investigation: J.R.S., R.A., B.A., S.Z., J.K., M.L.; Methodology: J.R.S., R.A., M.L.; Project administration: M.L.; Resources: J.R.S., M.L.; Software: J.R.S.; Supervision: M.L.; Validation: J.R.S.; Visualization: J.R.S., B.A., M.L.; Writing - original draft: J.R.S., B.A., M.L.; Writing - review \& editing: J.R.S., M.L.

\section{Funding}

This work was supported partially by US Public Health Service Award from the National Institutes of Health (MH108956) with additional support from the College of Arts and Sciences, the Department of Biochemistry and Cellular and Molecular Biology, and the Office of Research at The University of Tennessee, Knoxville.

\section{References}

Adler, J and I Parmryd (2010). "Quantifying colocalization by correlation: the Pearson correlation coefficient is superior to the Mander's overlap coefficient." Cytometry A 77(8): 733-742. DOI: 10.1002/cyto.a.20896.

Adryan, B, G Woerfel, I Birch-Machin, S Gao, M Quick, L Meadows, S Russell and R White (2007). "Genomic mapping of Suppressor of Hairy-wing binding sites in Drosophila." Genome Biol 8(8): R167. DOI: 10.1186/gb-2007-8-8-r167.

Afgan, E, D Baker, B Batut, M van den Beek, D Bouvier, M Cech, J Chilton, D Clements, N Coraor, BA Gruning, A Guerler, J HillmanJackson, S Hiltemann, V Jalili, H Rasche, N Soranzo, J Goecks, J Taylor, A Nekrutenko and D Blankenberg (2018). "The Galaxy platform for accessible, reproducible and collaborative biomedical analyses: 2018 update." Nucleic Acids Res 46(W1): W537-W544. DOI: $10.1093 /$ nar/gky379.

Alekseyenko, AA, AA Gorchakov, BM Zee, SM Fuchs, PV Kharchenko and MI Kuroda (2014). "Heterochromatin-associated interactions of Drosophila HP1a with dADD1, HIPP1, and repetitive RNAs." Genes Dev 28(13): 1445-1460. DOI: 10.1101/gad.241950.114.

Bag, I, RK Dale, C Palmer and EP Lei (2019). "The zinc-finger protein CLAMP promotes gypsy chromatin insulator function in Drosophila (doi:10.1242/jcs.226092)." J Cell Sci 132(6). DOI: $10.1242 /$ jcs. 231977 .

Baldi, S and PB Becker (2013). "The variant histone H2A.V of Drosophila--three roles, two guises." Chromosoma 122(4): 245258. DOI: 10.1007/s00412-013-0409-x.

Baldi, S, DS Jain, L Harpprecht, A Zabel, M Scheibe, F Butter, T Straub and PB Becker (2018). "Genome-wide Rules of Nucleosome Phasing in Drosophila." Mol Cell 72(4): 661-672 e664. DOI: 10.1016/j.molcel.2018.09.032.
Baxley, RM, JD Bullard, MW Klein, AG Fell, JA Morales-Rosado, T Duan and PK Geyer (2017). "Deciphering the DNA code for the function of the Drosophila polydactyl zinc finger protein Suppressor of Hairy-wing." Nucleic Acids Res 45(8): 4463-4478. DOI: 10.1093/nar/gkx040.

Baxley, RM, AA Soshnev, DE Koryakov, IF Zhimulev and PK Geyer (2011). "The role of the Suppressor of Hairy-wing insulator protein in Drosophila oogenesis." Dev Biol 356(2): 398-410. DOI: 10.1016/j.ydbio.2011.05.666.

Bell, AC, GG West and G Felsenfeld (1999). "The Protein CTCF Is Required for the Enhancer Blocking Activity of Vertebrate Insulators." Cell 98(3): 387 - 396. DOI: 10.1016/s00928674(00)81967-4.

Bialojan, C and A Takai (1988). "Inhibitory effect of a marinesponge toxin, okadaic acid, on protein phosphatases. Specificity and kinetics." Biochemical Journal 256(1): 283-290. DOI: 10.1042/bj2560283.

Bonchuk, A, O Maksimenko, O Kyrchanova, T Ivlieva, V Mogila, G Deshpande, D Wolle, P Schedl and P Georgiev (2015). "Functional role of dimerization and CP190 interacting domains of CTCF protein in Drosophila melanogaster." BMC Biol 13: 63. DOI: 10.1186/s12915-015-0168-7.

Bushey, AM, E Ramos and VG Corces (2009). "Three subclasses of a Drosophila insulator show distinct and cell type-specific genomic distributions." Genes Dev 23(11): 1338-1350. DOI: 10.1101/gad.1798209.

Cai, HN and M Levine (1997). "The gypsy insulator can function as a promoter-specific silencer in the Drosophila embryo." The EMBO Journal 16: 1732-1741. DOI: 10.1093/emboj/16.7.1732.

Canela, A, Y Maman, S Jung, N Wong, E Callen, A Day, KR KiefferKwon, A Pekowska, H Zhang, SSP Rao, SC Huang, PJ McKinnon, PD Aplan, Y Pommier, EL Aiden, R Casellas and A Nussenzweig (2017). "Genome Organization Drives Chromosome Fragility." Cell 170(3): 507-521 e518. DOI: 10.1016/j.cell.2017.06.034.

Chen, D, M Brovkina, LH Matzat and EP Lei (2019). "Shep RNABinding Capacity Is Required for Antagonism of gypsy Chromatin Insulator Activity." G3 (Bethesda) 9(3): 749-754. DOI: 10.1534/g3.118.200923.

Chen, Y, N Negre, Q Li, JO Mieczkowska, M Slattery, T Liu, Y Zhang, T-K Kim, HH He, J Zieba, Y Ruan, PJ Bickel, RM Myers, BJ Wold, KP White, JD Lieb and XS Liu (2012). "Systematic evaluation of factors influencing ChIP-seq fidelity." Nature methods 9(6): 609-614. DOI: 10.1038/nmeth.1985.

Chowdhury, D, MC Keogh, H Ishii, CL Peterson, S Buratowski and J Lieberman (2005). "gamma-H2AX dephosphorylation by protein phosphatase 2A facilitates DNA double-strand break repair." Mol Cell 20(5): 801-809. DOI: 10.1016/j.molcel.2005.10.003.

Costes, SV, D Daelemans, EH Cho, Z Dobbin, G Pavlakis and S Lockett (2004). "Automatic and quantitative measurement of protein-protein colocalization in live cells." Biophys J 86(6): 39934003. DOI: 10.1529/biophysj.103.038422. 
Docquier, F, D Farrar, V D'Arcy, I Chernukhin, AF Robinson, D Loukinov, S Vatolin, S Pack, A Mackay, RA Harris, H Dorricott, MJ O'Hare, V Lobanenkov and E Klenova (2005). "Heightened Expression of CTCF in Breast Cancer Cells Is Associated with Resistance to Apoptosis." 65(12): 5112-5122. DOI: 10.1158/00085472.CAN-03-3498 \%J Cancer Research.

Duan, T and PK Geyer (2018). "Spermiogenesis and Male Fertility Require the Function of Suppressor of Hairy-Wing in Somatic Cyst Cells of Drosophila." Genetics 209(3): 757-772. DOI: 10.1534/genetics.118.301088.

Dunn, KW, MM Kamocka and JH McDonald (2011). "A practical guide to evaluating colocalization in biological microscopy." Am J Physiol Cell Physiol 300(4): C723-742. DOI: 10.1152/ajpcell.00462.2010.

Fudenberg, G, M Imakaev, C Lu, A Goloborodko, N Abdennur and LA Mirny (2016). "Formation of Chromosomal Domains by Loop Extrusion." Cell Rep 15(9): 2038-2049. DOI: 10.1016/j.celrep.2016.04.085.

Fujioka, M, G Sun and JB Jaynes (2013). "The Drosophila eve insulator Homie promotes eve expression and protects the adjacent gene from repression by polycomb spreading." PLOS Genet 9(10): e1003883. DOI: 10.1371/journal.pgen.1003883.

Gause, M, P Morcillo and D Dorsett (2001). "Insulation of enhancer-promoter communication by a gypsy transposon insert in the Drosophila cut gene: cooperation between suppressor of hairy-wing and modifier of mdg4 proteins." Mol Cell Biol 21(14): 4807-4817. DOI: 10.1128/MCB.21.14.4807-4817.2001.

Gdula, DA, TI Gerasimova and VG Corces (1996). "Genetic and molecular analysis of the gypsy chromatin insulator of Drosophila." Proc Natl Acad Sci U S A 93: 9378-9383.

Georgiev, P and M Kozycina (1996). "Interaction Between Mutations in the suppressor of Hairy wing and modifier of mdg4 Genes of Drosophila melanogaster Affecting the Phenotype of gypsy-Induced Mutations." Genetics 142(2): 425-436.

Gerasimova, TI, K Byrd and VG Corces (2000). "A Chromatin Insulator Determines the Nuclear Localization of DNA." Molecular Cell 6(5): 1025-1035. DOI: 10.1016/S1097-2765(00)00101-5.

Gerasimova, TI and VG Corces (1998). "Polycomb and trithorax group proteins mediate the function of a chromatin insulator." Cell 92: 511 - 521. DOI: 10.1016/s0092-8674(00)80944-7.

Geyer, PK and VG Corces (1992). "DNA position-specific repression of transcription by a Drosophila zinc finger protein." Genes \& Development 6: 1865-1873.

Ghosh, D, TI Gerasimova and VG Corces (2001). "Interactions between the $\mathrm{Su}(\mathrm{Hw})$ and $\mathrm{Mod}(\mathrm{mdg} 4)$ proteins required for gypsy insulator function." The EMBO Journal 20: 2518-2527. DOI: 10.1093/emboj/20.10.2518.

Giaimo, BD, F Ferrante, A Herchenrother, SB Hake and T Borggrefe (2019). "The histone variant H2A.Z in gene regulation." Epigenetics Chromatin 12(1): 37. DOI: 10.1186/s13072-019-0274-9.
Glenn, SE and PK Geyer (2019). "Investigation of the Developmental Requirements of Drosophila HP1 and Insulator Protein Partner, HIPP1." G3 (Bethesda) 9(2): 345-357. DOI: 10.1534/g3.118.200705.

Golovnin, A, L Melnikova, I Shapovalov, M Kostyuchenko and P Georgiev (2015). "EAST Organizes Drosophila Insulator Proteins in the Interchromosomal Nuclear Compartment and Modulates CP190 Binding to Chromatin." PLoS One 10(10): e0140991. DOI: 10.1371/journal.pone.0140991.

Golovnin, A, I Volkov and P Georgiev (2012). "SUMO conjugation is required for the assembly of Drosophila $\mathrm{Su}(\mathrm{Hw})$ and $\mathrm{Mod}(\mathrm{mdg} 4)$ into insulator bodies that facilitate insulator complex formation." J Cell Sci 125(Pt 8): 2064-2074. DOI: 10.1242/jcs.100172.

Golovnin, AK, VV Molodina, PG Georgiev and LS Melnikova (2016). "Zinc finger domain of $\mathrm{Su}(\mathrm{Hw})$ protein is required for the formation of functional $\mathrm{Su}(\mathrm{Hw})$-dependent insulator complex." Dokl Biochem Biophys 469(1): 247-252. DOI: 10.1134/S1607672916040049.

Grigorian, M, H DeBruhl and JS Lipsick (2017). "The role of variant histone H2AV in Drosophila melanogaster larval hematopoiesis." Development 144(8): 1441-1449. DOI: 10.1242/dev.142729.

Guerrero, PA and KA Maggert (2011). "The CCCTC-binding factor (CTCF) of Drosophila contributes to the regulation of the ribosomal DNA and nucleolar stability." PLoS One 6(1): e16401. DOI: 10.1371/journal.pone.0016401.

Guo, YA, MM Chang, W Huang, WF Ooi, M Xing, P Tan and AJ Skanderup (2018). "Mutation hotspots at CTCF binding sites coupled to chromosomal instability in gastrointestinal cancers." Nat Commun 9(1): 1520. DOI: 10.1038/s41467-018-03828-2.

Han, D, Q Chen, J Shi, F Zhang and X Yu (2017). "CTCF participates in DNA damage response via poly(ADP-ribosyl)ation." Sci Rep 7: 43530. DOI: 10.1038/srep43530.

Harrison, DA, DA Gdula, RS Coyne and VG Corces (1993). "A Leucine Zipper Domain of the Suppressor of Hairy-wing Protein Mediates Its Repressive Effect on Enhancer Function." Genes \& Development 7(10): 1966-1978.

Harrison, DA, PK Geyer, C Spana and VG Corces (1989). "The gypsy retrotransposon of Drosophila melanogaster: mechanisms of mutagenesis and interaction with the suppressor of Hairy-wing locus." Dev Genet 10(3): 239-248. DOI: 10.1002/dvg.1020100313.

Harrison, DA, MA Mortin and VG Corces (1992). "The RNA polymerase II 15-kilodalton subunit is essential for viability in Drosophila melanogaster." Molecular and Cellular Biology 12(3): 928-935. DOI: 10.1128/mcb.12.3.928.

Hartenstein, V and JW Posakony (1989). "Development of adult sensilla on the wing and notum of Drosophila melanogaster." Development 107(2): 389-405.

Haystead, TAJ, ATR Sim, D Carling, RC Honnor, Y Tsukitani, P Cohen and DG Hardie (1989). "Effects of the tumour promoter okadaic acid on intracellular protein phosphorylation and metabolism." Nature 337(6202): 78-81. DOI: 10.1038/337078a0. 
Heger, $\mathbf{P}$ and $\mathbf{T}$ Wiehe (2014). "New tools in the box: an evolutionary synopsis of chromatin insulators." Trends Genet 30(5): 161-171. DOI: 10.1016/j.tig.2014.03.004.

Hilmi, K, M Jangal, M Marques, T Zhao, A Saad, C Zhang, VM Luo, A Syme, C Rejon, Z Yu, A Krum, MR Rabian, S Richard, M AlaouiJamali, A Orthwein, L McCaffrey and M Witcher (2017). "CTCF facilitates DNA double-strand break repair by enhancing homologous recombination repair." Science Advances 3(e1601898): 1-14.

Honkanen, RE and T Golden (2002). "Regulators of Serine Threonine Protein Phosphatases at the Dawn of a Clinical Era." Current Medicinal Chemistry 9: 2055-2075. DOI: 10.2174/0929867023368836.

Hoskins, RA, JW Carlson, KH Wan, S Park, I Mendez, SE Galle, BW Booth, BD Pfeiffer, RA George, R Svirskas, M Krzywinski, J Schein, MC Accardo, E Damia, G Messina, M Mendez-Lago, B de Pablos, OV Demakova, EN Andreyeva, LV Boldyreva, M Marra, AB Carvalho, P Dimitri, A Villasante, IF Zhimulev, GM Rubin, GH Karpen and SE Celniker (2015). "The Release 6 reference sequence of the Drosophila melanogaster genome." Genome Res 25(3): 445458. DOI: 10.1101/gr.185579.114.

Hsu, S-J, EC Stow, JR Simmons, HA Wallace, AM Lopez, S Stroud and $M$ Labrador (2019). "Mutations in the Insulator Protein Suppressor of Hairy Wing Induce Genome Instability." bioRxiv. DOI: $10.1101 / 551002$.

Hsu, SJ, MP Plata, B Ernest, S Asgarifar and M Labrador (2015). "The insulator protein Suppressor of Hairy wing is required for proper ring canal development during oogenesis in Drosophila." Dev Biol 403(1): 57-68. DOI: 10.1016/j.ydbio.2015.03.024.

Hsu, SJ, EC Stow, JR Simmons, HA Wallace, AM Lopez, S Stroud and M Labrador (2020). "Mutations in the insulator protein Suppressor of Hairy wing induce genome instability." Chromosoma 129(3-4): 255-274. DOI: 10.1007/s00412-020-00743-8.

Jack, J, D Dorsett, Y Delotto and S Liu (1991). "Expression of the cut locus in the Drosophila wing margin is required for cell type specification and is regulated by a distant enhancer." Development 113: $735-747$.

Jack, JW (1985). "Molecular organization of the cut locus of drosophila melanogaster." Cell 42(3): 869-876. DOI: 10.1016/00928674(85)90283-1.

Jox, T, MK Buxa, D Bohla, I Ullah, I Mačinković, A Brehm, M Bartkuhn and R Renkawitz (2017). "Drosophila CP190- and dCTCFmediated enhancer blocking is augmented by SUMOylation." Epigenetics \& Chromatin 10(1): 32. DOI: 10.1186/s13072-0170140-6.

Joyce, EF, M Pedersen, S Tiong, SK White-Brown, A Paul, SD Campbell and KS McKim (2011). "Drosophila ATM and ATR have distinct activities in the regulation of meiotic DNA damage and repair." J Cell Biol 195(3): 359-367. DOI: 10.1083/jcb.201104121.

Kellum, R and P Schedl (1991). "A position-effect assay for boundaries of higher order chromosomal domains." Cell 64: 941950.
Kemp, CJ, JM Moore, R Moser, B Bernard, M Teater, LE Smith, NA Rabaia, KE Gurley, J Guinney, SE Busch, R Shaknovich, VV Lobanenkov, D Liggitt, I Shmulevich, A Melnick and GN Filippova (2014). "CTCF haploinsufficiency destabilizes DNA methylation and predisposes to cancer." Cell Rep 7(4): 1020-1029. DOI: 10.1016/j.celrep.2014.04.004.

Kim, J, B Shen, C Rosen and D Dorsett (1996). "The DNA-binding and enhancer-blocking domains of the Drosophila suppressor of Hairy-wing protein." Molecular and Cellular Biology 16(7): 33813392.

King, MR, LH Matzat, RK Dale, SJ Lim and EP Lei (2014). "The RNAbinding protein Rumpelstiltskin antagonizes gypsy chromatin insulator function in a tissue-specific manner." J Cell Sci 127(Pt 13): 2956-2966. DOI: 10.1242/jcs.151126.

Klug, WS, D Bodenstein and RC King (1968). "Oogenesis in the suppressor2 of Hairy-wing mutant of Drosophila melanogaster." Journal of Experiemental Zoology 167: 151-156.

Kotova, E, N Lodhi, M Jarnik, AD Pinnola, Y Ji and AV Tulin (2011). "Drosophila histone H2A variant (H2Av) controls poly(ADP-ribose) polymerase 1 (PARP1) activation in chromatin." Proc Natl Acad Sci U S A 108(15): 6205-6210. DOI: 10.1073/pnas.1019644108.

Kuhn-Parnell, EJ, C Helou, DJ Marion, BL Gilmore, TJ Parnell, MS Wold and PK Geyer (2008). "Investigation of the properties of nongypsy suppressor of hairy-wing-binding sites." Genetics 179(3): 1263-1273. DOI: 10.1534/genetics.108.087254.

Labrador, M and VG Corces (2002). "Setting the Boundaries of Chromatin Domains and Nuclear Organization." Cell 111(2): 151 154. DOI: 10.1016/S0092-8674(02)01004-8.

Lake, CM, JK Holsclaw, SP Bellendir, J Sekelsky and RS Hawley (2013). "The development of a monoclonal antibody recognizing the Drosophila melanogaster phosphorylated histone $\mathrm{H} 2 \mathrm{~A}$ variant (gamma-H2AV)." G3 (Bethesda) 3(9): 1539-1543. DOI: 10.1534/g3.113.006833.

Lang, F, X Li, W Zheng, Z Li, D Lu, G Chen, D Gong, L Yang, J Fu, P Shi and J Zhou (2017). "CTCF prevents genomic instability by promoting homologous recombination-directed DNA doublestrand break repair." Proc Natl Acad Sci U S A 114(41): 1091210917. DOI: 10.1073/pnas.1704076114.

Langmead, B and SL Salzberg (2012). "Fast gapped-read alignment with Bowtie 2." Nat Methods 9(4): 357-359. DOI: 10.1038/nmeth.1923.

Lankenau, DH, MV Peluso and S Lankenau (2000). "The Su(Hw) chromatin insulator protein alters double-strand break repair frequencies in the Drosophila germ line." Chromosoma 109(1-2): 148-160. DOI: 10.1007/s004120050423.

LaRocque, JR, B Jaklevic, TT Su and J Sekelsky (2007). "Drosophila ATR in double-strand break repair." Genetics 175(3): 1023-1033. DOI: 10.1534/genetics.106.067330.

Li, Y, RL Armstrong, RJ Duronio and DM MacAlpine (2016). "Methylation of histone $\mathrm{H} 4$ lysine 20 by PR-Set7 ensures the integrity of late replicating sequence domains in Drosophila." Nucleic Acids Res 44(15): 7204-7218. DOI: 10.1093/nar/gkw333. 
Li, Z, MR Johnson, Z Ke, L Chen and MA Welte (2014). "Drosophila lipid droplets buffer the H2Av supply to protect early embryonic development." Curr Biol 24(13): 1485-1491. DOI: 10.1016/j.cub.2014.05.022.

Liu, S, E McLeod and J Jack (1991). "Four distinct regulatory regions of the cut locus and their effect on cell type specification in Drosophila." Genetics 127(1): 151-159.

Macurek, L, A Lindqvist, O Voets, J Kool, HR Vos and RH Medema (2010). "Wip1 phosphatase is associated with chromatin and dephosphorylates gammaH2AX to promote checkpoint inhibition." Oncogene 29(15): 2281-2291. DOI: 10.1038/onc.2009.501.

Madigan, JP, HL Chotkowski and RL Glaser (2002). "DNA doublestrand break-induced phosphorylation of Drosophila histone variant $\mathrm{H} 2 \mathrm{Av}$ helps prevent radiation-induced apoptosis." Nucleic Acids Res 30(17): 3698 - 3705. DOI: 10.1093/nar/gkf496.

Manders, EMM, J Stap, GJ Brakenhoff, R Van Driel and JA Aten (1992). "Dynamics of three-dimensional replication patterns during the S-phase, analysed by double labelling of DNA and confocal microscopy." J Cell Sci 103: 857-862.

Manders, EMM, FJ Verbeek and JA Aten (1993). "Measurement of co-localization of objects in dual-color confocal images." Journal of Microscopy 169: 375-382.

Matzat, LH, RK Dale, N Moshkovich and EP Lei (2012). "Tissuespecific regulation of chromatin insulator function." PLoS Genet 8(11): e1003069. DOI: 10.1371/journal.pgen.1003069.

Melnikova, L, M Kostyuchenko, V Molodina, A Parshikov, P Georgiev and A Golovnin (2017). "Multiple interactions are involved in a highly specific association of the $\operatorname{Mod}(\operatorname{mdg} 4)-67.2$ isoform with the $\mathrm{Su}(\mathrm{Hw})$ sites in Drosophila." Open Biol 7(10). DOI: 10.1098/rsob.170150.

Melnikova, L, M Kostyuchenko, V Molodina, A Parshikov, P Georgiev and A Golovnin (2018). "Interactions between BTB domain of CP190 and two adjacent regions in $\mathrm{Su}(\mathrm{Hw})$ are required for the insulator complex formation." Chromosoma 127(1): 59-71. DOI: 10.1007/s00412-017-0645-6.

Melnikova, L, M Kostyuchenko, A Parshikov, P Georgiev and A Golovnin (2018). "Role of $\mathrm{Su}(\mathrm{Hw})$ zinc finger 10 and interaction with CP190 and Mod(mdg4) proteins in recruiting the $\mathrm{Su}(\mathrm{Hw})$ complex to chromatin sites in Drosophila." PLoS One 13(2): e0193497. DOI: 10.1371/journal.pone.0193497.

Melnikova, L, V Molodina, M Erokhin, P Georgiev and A Golovnin (2019). "HIPP1 stabilizes the interaction between CP190 and $\mathrm{Su}(\mathrm{Hw})$ in the Drosophila insulator complex." Sci Rep 9(1): 19102. DOI: 10.1038/s41598-019-55617-6.

Melnikova, L, I Shapovalov, M Kostyuchenko, P Georgiev and A Golovnin (2017). "EAST affects the activity of Su(Hw) insulators by two different mechanisms in Drosophila melanogaster." Chromosoma 126(2): 299-311. DOI: 10.1007/s00412-016-0596-3.

Merigliano, C, A Marzio, F Renda, MP Somma, M Gatti and F Verni (2017). "A Role for the Twins Protein Phosphatase (PP2A-B55) in the Maintenance of Drosophila Genome Integrity." Genetics 205(3): 1151-1167. DOI: 10.1534/genetics.116.192781.
Mohan, M, M Bartkuhn, M Herold, A Philippen, N Heinl, I Bardenhagen, J Leers, RA White, R Renkawitz-Pohl, H Saumweber and R Renkawitz (2007). "The Drosophila insulator proteins CTCF and CP190 link enhancer blocking to body patterning." EMBO J 26(19): 4203-4214. DOI: 10.1038/sj.emboj.7601851.

Mongelard, F, M Labrador, EM Baxter, TI Gerasimova and VG Corces (2002). "Trans-splicing as a Novel Mechanism to Explain Interallelic Complementation in Drosophila." Genetics 160(4): 1481-1487.

Morillo Prado, JR, S Srinivasan and MT Fuller (2013). "The histone variant His2Av is required for adult stem cell maintenance in the Drosophila testis." PLoS Genet 9(11): e1003903. DOI: 10.1371/journal.pgen.1003903.

Nakada, S, GI Chen, AC Gingras and D Durocher (2008). "PP4 is a gamma $\mathrm{H} 2 \mathrm{AX}$ phosphatase required for recovery from the DNA damage checkpoint." EMBO Rep 9(10): 1019-1026. DOI: 10.1038/embor.2008.162.

Natale, F, A Rapp, W Yu, A Maiser, H Harz, A Scholl, S Grulich, T Anton, D Horl, W Chen, M Durante, G Taucher-Scholz, H Leonhardt and MC Cardoso (2017). "Identification of the elementary structural units of the DNA damage response." Nat Commun 8: 15760 . DOI: 10.1038/ncomms15760.

Negre, N, CD Brown, PK Shah, P Kheradpour, CA Morrison, JG Henikoff, X Feng, K Ahmad, S Russell, RA White, L Stein, S Henikoff, M Kellis and KP White (2010). "A comprehensive map of insulator elements for the Drosophila genome." PLoS Genet 6(1): e1000814. DOI: 10.1371/journal.pgen.1000814.

Ooegema, K, WGF Whitfield and B Alberts (1995). "The cell cycledependent localization of the CP190 centrosomal protein is determined by the coordinate action of two separable domains." Journal of Cell Biology 131(5): 1261-1273.

Ozdemir, I and MC Gambetta (2019). "The Role of Insulation in Patterning Gene Expression." Genes (Basel) 10(10). DOI: 10.3390/genes10100767

Pai, CY, EP Lei, D Ghosh and VG Corces (2004). "The centrosomal protein CP190 is a component of the gypsy chromatin insulator." Mol Cell 16(5): 737-748. DOI: 10.1016/j.molcel.2004.11.004.

Pearson, K (1895). "Mathematical contributions to the theory of evolution." Philosophical Transactions of the Royal Society of London. Series A, Containing Papers of a Mathematical or Physical Character(187): 253 - 318. DOI: 10.1098/rsta.1896.0007.

Postika, N, M Metzler, M Affolter, M Müller, P Schedl, P Georgiev and $O$ Kyrchanova (2018). "Boundaries mediate long-distance interactions between enhancers and promoters in the Drosophila Bithorax complex." PLoS Genet 14(12): e1007702. DOI: 10.1371/journal.pgen.1007702.

Ramirez, F, V Bhardwaj, L Arrigoni, KC Lam, BA Gruning, J Villaveces, B Habermann, A Akhtar and T Manke (2018). "Highresolution TADs reveal DNA sequences underlying genome organization in flies." Nat Commun 9(1): 189. DOI: 10.1038/s41467017-02525-w. 
Rao, Suhas SP, Miriam H Huntley, Neva C Durand, Elena K Stamenova, Ivan D Bochkov, James T Robinson, Adrian L Sanborn, I Machol, Arina D Omer, Eric S Lander and Erez L Aiden (2014). "A 3D Map of the Human Genome at Kilobase Resolution Reveals Principles of Chromatin Looping." Cell 159(7): 1665-1680. DOI: 10.1016/j.cell.2014.11.021.

Robinson, JT, H Thorvaldsdóttir, W Winckler, M Guttman, ES Lander, G Getz and JP Mesirov (2011). "Integrative genomics viewer." Nature Biotechnology 29(1): 24-26. DOI: 10.1038/nbt.1754.

Rogakou, EP, DR Pilch, AH Orr, VS Ivanova and WM Bonner (1998). "DNA Double-stranded Breaks Induce Histone H2AX Phosphorylation on Serine 139." The Journal of Biological Chemistry 273: 5858-5868. DOI: 10.1074/jbc.273.10.5858.

Sanders, JT, TF Freeman, Y Xu, R Golloshi, MA Stallard, AM Hill, R San Martin, AS Balajee and RP McCord (2020). "Radiation-induced DNA damage and repair effects on 3D genome organization." Nat Commun 11(1): 6178. DOI: 10.1038/s41467-020-20047-w.

Schindelin, J, I Arganda-Carreras, E Frise, V Kaynig, M Longair, T Pietzsch, S Preibisch, C Rueden, S Saalfeld, B Schmid, JY Tinevez, DJ White, V Hartenstein, K Eliceiri, P Tomancak and A Cardona (2012). "Fiji: an open-source platform for biological-image analysis." Nat Methods 9(7): 676-682. DOI: 10.1038/nmeth.2019.

Schoborg, T and M Labrador (2014). "Expanding the roles of chromatin insulators in nuclear architecture, chromatin organization and genome function." Cell Mol Life Sci 71(21): 40894113. DOI: 10.1007/s00018-014-1672-6.

Schoborg, T, R Rickels, J Barrios and M Labrador (2013). "Chromatin insulator bodies are nuclear structures that form in response to osmotic stress and cell death." J Cell Biol 202(2): 261276. DOI: $10.1083 /$ jcb.201304181.

Sirbu, BM and D Cortez (2013). "DNA damage response: three levels of DNA repair regulation." Cold Spring Harb Perspect Biol 5(8): a012724. DOI: 10.1101/cshperspect.a012724.

Soshnev, AA, RM Baxley, JR Manak, K Tan and PK Geyer (2013). "The insulator protein Suppressor of Hairy-wing is an essential transcriptional repressor in the Drosophila ovary." Development 140(17): 3613-3623. DOI: 10.1242/dev.094953.

Soshnev, AA, B He, RM Baxley, N Jiang, CM Hart, K Tan and PK Geyer (2012). "Genome-wide studies of the multi-zinc finger Drosophila Suppressor of Hairy-wing protein in the ovary." Nucleic Acids Res 40(12): 5415-5431. DOI: 10.1093/nar/gks225.

Stiff, T, M O'Driscoll, N Rief, $\mathrm{K}$ Iwabuchi, M Lobrich and PA Jeggo (2004). "ATM and DNA-PK Function Redundantly to Phosphorylate H2AX after Exposure to lonizing Radiation." Cancer Research 64(7): 2390-2396.

van Berkum, NL, E Lieberman-Aiden, L Williams, M Imakaev, A Gnirke, LA Mirny, J Dekker and ES Lander (2010). "Hi-C: A Method to Study the Three-dimensional Architecture of Genomes." JoVE(39): e1869. DOI: doi:10.3791/1869.

Van Bortle, K, MH Nichols, C Ong, N Takenaka, ZS Qin and VG Corces (2014). "Insulator function and topological domain border strength scale with architectural protein occupancy." Genome Biology 15(R82): 1-18.

van Daal, A and SCR Elgin (1992). "A histone variant, H2AvD, is essential in Drosophila melanogaster." Molecular Biology of the Cell 3: 593-602. DOI: 10.1091/mbc.3.6.593.

van Daal, A, EM White, MA Gorovsky and CR Elgin (1988). "Drosophila has a single copy of the gene encoding a highly conserved histone H2A variant of the H2A. F/Z type." Nucleic Acids Research 16(15): 7487-7497. DOI: 10.1093/nar/16.15.7487.

Wallace, HA, MP Plata, HJ Kang, M Ross and M Labrador (2010). "Chromatin insulators specifically associate with different levels of higher-order chromatin organization in Drosophila." Chromosoma 119(2): 177-194. DOI: 10.1007/s00412-009-0246-0.

Ward, IM and J Chen (2001). "Histone H2AX is phosphorylated in an ATR-dependent manner in response to replicational stress." J Biol Chem 276(51): 47759-47762. DOI: 10.1074/jbc.C100569200.

Weber, CM, JG Henikoff and S Henikoff (2010). "H2A.Z nucleosomes enriched over active genes are homotypic." Nat Struct Mol Biol 17(12): 1500-1507. DOI: 10.1038/nsmb.1926.

Wen, Z, L Zhang, H Ruan and G Li (2020). "Histone variant H2A.Z regulates nucleosome unwrapping and CTCF binding in mouse ES cells." Nucleic Acids Res 48(11): 5939-5952. DOI: 10.1093/nar/gkaa360.

Wittkopp, PJ, JR True and SB Carroll (2002). "Reciprocal functions of the Drosophila Yellow and Ebony proteins in the development and evolution of pigment patterns." Development 129(8): 18491858.

Ye, T, AR Krebs, MA Choukrallah, C Keime, F Plewniak, I Davidson and L Tora (2011). "seqMINER: an integrated ChIP-seq data interpretation platform." Nucleic Acids Res 39(6): e35. DOI: 10.1093/nar/gkq1287.

Zhan, $\mathbf{X}$ and DJ Liu (2015). "SEQMINER: An R-Package to Facilitate the Functional Interpretation of Sequence-Based Associations." Genetic Epidemiology 39(8): 619-623. DOI: doi.org/10.1002/gepi.21918.

Zhang, Y, T Liu, CA Meyer, J Eeckhoute, DS Johnson, BE Bernstein, C Nusbaum, RM Myers, M Brown, W Li and XS Liu (2008). "Modelbased analysis of ChIP-Seq (MACS)." Genome Biol 9(9): R137. DOI: 10.1186/gb-2008-9-9-r137. 